\title{
Integrating the Pillared-Layer Strategy and Pore-Space Partition Method to Construct Multicomponent MOFs for $\mathrm{C}_{2} \mathrm{H}_{2} / \mathrm{CO}_{2}$ Separation
}

Lizhen Liu, ${ }^{\dagger, \star}, \S$ Zizhu Yao, ${ }^{\dagger, \S}$ Yingxiang Ye, ${ }^{\dagger}$ Yike Yang, ${ }^{\dagger}$ Quanjie Lin, ${ }^{\dagger}$ Zhangjing Zhang, ${ }^{*}{ }^{\dagger}$ Michael O'Keeffe ' and Shengchang Xiang* ${ }^{\dagger}$

$\dagger$ Fujian Provincial Key Laboratory of Polymer Materials, College of Chemistry and Materials Science, Fujian Normal University, 32 Shangsan Road, Fuzhou 350007, P. R. China

$\$$ College of Materials Science and Engineering, Key Laboratory of Polymer Materials and Products of Universities in Fujian, Fujian University of Technology, Fuzhou, Fujian 350118, P.R. China.

\| School of Molecular Sciences, Arizona State University, Tempe, Arizona 85287, United States

$\S$ L. Liu and Z. Yao contributed equally.

Correspondence and requests for materials should be addressed to S.X (scxiang@fjnu.edu.cn) and Z.Z. (zzhang@fjnu.edu.cn). 


\section{Contents}

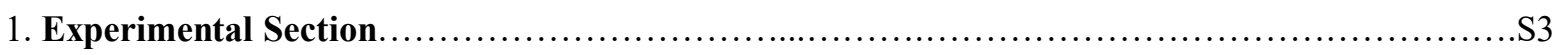

2. Table S1 X-ray crystallography: summary of data collection and refinement details............................S9

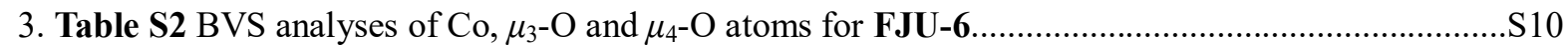

4. Figure S1 Coordination environments of trinuclear $\left[\mathrm{Co}_{3}\left(\mu_{3}-\mathrm{OH}\right) \mathrm{N}_{3}(\mathrm{COO})_{6}\right]$ cluster and hexanuclear $\left[\mathrm{Co}_{6}\left(\mu_{4}-\mathrm{O}\right)_{2} \mathrm{~N}_{4}(\mathrm{COO})_{8}\right]$ cluster in FJU-6

5. Figure S2 Pore space partition through symmetry-matching regulated ligand BTB insertion, which form cage-II and square channels in FJU-6 along the $c$ axis. S11

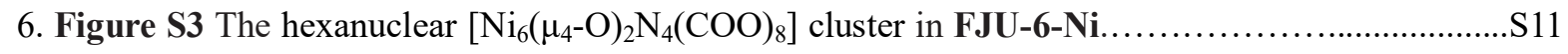

7. Figure S4 Taking cage as node, llz net can be obtained by modifying sqp net with PSP............. S12

8. Figure S5 PXRD patterns of eight mm-MOFs after heated at different temperatures..................S13

9. Figure S6 Illustration of the in-situ activated PXRD device..................................... S14

10. Figure S7 PXRD patterns of eight mm-MOFs after vacuum in situ activation for $12 \mathrm{~h} . \ldots \ldots \ldots \ldots . . . . . .515$

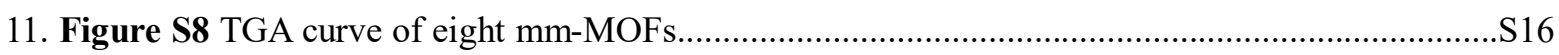

12. Figure S9 Systematic modulation of the ligands and metals of FJU-6 to produce mm-MOFs.......S17

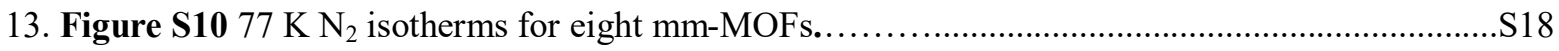

14. Figure $\mathbf{S 1 1} 77 \mathrm{~K} \mathrm{~N}_{2}$ isotherms of FJU-6-TATB at different activation times.................................... 18

15. Figure S12 Pore size distribution plots for eight mm-MOFs.................................. 19

16. Figure S13 Adsorption isotherms for $\mathrm{C}_{2} \mathrm{H}_{2}$ and $\mathrm{CO}_{2}$ of FJU-6 and FJU-6-PTB at $296 \mathrm{~K} \ldots \ldots \ldots . . . \mathrm{S} 19$

17. Figure S14 Adsorption isotherms for $\mathrm{C}_{2} \mathrm{H}_{2}$ of FJU-6, FJU-6-PTB, and FJU-6-TATB at $273 \mathrm{~K}$.....S20

18. Figure $\mathbf{S 1 5}$ The plots of virial equation of $\mathrm{C}_{2} \mathrm{H}_{2}$ and $\mathrm{CO}_{2}$ in FJU-6-TATB $\ldots \ldots \ldots \ldots \ldots \ldots \ldots . . . \ldots \ldots$

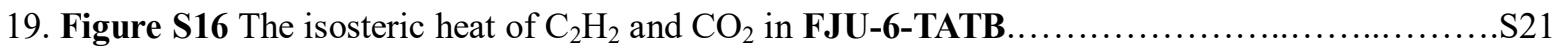

20. Figure S17 The graphs of the Single-site Langmuir-Freundlich equations fit for adsorption of $\mathrm{C}_{2} \mathrm{H}_{2}$

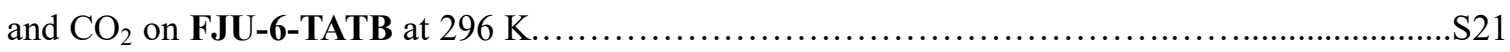

21. Table S3 Summary of MOFs with $\mathrm{C}_{2} \mathrm{H}_{2} / \mathrm{CO}_{2}$ (50/50) breakthrough performance and related gas uptake and separation values under ambient conditions

22. Figure $\mathbf{S 1 8}$ Comparison of $\mathrm{C}_{2} \mathrm{H}_{2} / \mathrm{CO}_{2}$ separation factor and $\mathrm{C}_{2} \mathrm{H}_{2}$ uptake at 1 bar in FJU-6-TATB,

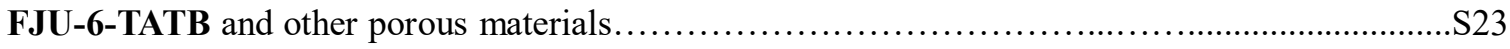

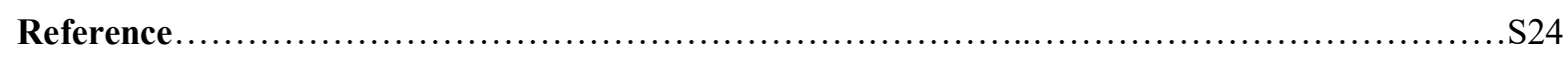




\section{Experimental Section}

\section{Materials and Methods}

All reagents and solvents used in synthetic studies are commercially available and were used as supplied without further purification. 1,3,5,-Tris(4-carboxyphenyl)benzene $\left(\mathrm{H}_{3} \mathrm{BTB}\right)$, 4,4',4"-s-triazine-2,4,6-triyltribenzoate ( H $_{3}$ TATB), isonicotinic acid (HINA), 3-methylisonicotinic acid (Me-HINA), 3-fluoroisonicotinic acid (F-HINA), 1,4-Naphthalenedicarboxlic acid $\left(\mathrm{H}_{2} \mathrm{NDC}\right)$, 1,4-dicarboxybenzene $\left(\mathrm{H}_{2} \mathrm{BDC}\right)$, and 2-Bromoterephthalic acid $\left(\mathrm{Br}-\mathrm{H}_{2} \mathrm{BDC}\right)$ were purchased from Tansoole. Powder X-ray diffraction (PXRD) was carried out with a PANalytical $\mathrm{X}^{\prime} \mathrm{Pert}^{3}$ powder diffractometer equipped with a $\mathrm{Cu}$ sealed tube $(\lambda=1.541874 \AA)$ at $40 \mathrm{kV}$ and $40 \mathrm{~mA}$ over the $2 \theta$ range of $5-30^{\circ}$. The simulated pattern was produced using the Mercury V1.4 program and single-crystal diffraction data. Elemental analyses $(\mathrm{C}, \mathrm{H}$ and $\mathrm{N})$ were performed on a Perkin-Elmer 240C analyzer. Thermal analysis was carried out on a METTLER TGA/SDTA 851 thermal analyzer from 30 to $600{ }^{\circ} \mathrm{C}$ at a heating rate of $10{ }^{\circ} \mathrm{C} \mathrm{min}^{-1}$ under $\mathrm{N}_{2}$ flow.

\section{Single-crystal X-ray structural analysis}

Data collection and structural analysis of eight mm-MOFs were performed on an Agilent Technologies SuperNova single crystal diffractometer equipped with graphite monochromatic Mo Ka radiation $(\lambda=0.71073 \AA)$ or $\mathrm{Cu}$ Ka radiation $(\lambda=1.54184 \AA)$. The crystal was kept at $100 \mathrm{~K}$ or $298 \mathrm{~K}$ during data collection. Using Olex $2^{1}$, the structure was solved with the Superflip $^{2}$ structure solution program using charge flipping and refined with the ShelXL ${ }^{3}$ refinement package using least squares minimization. All nonhydrogen atoms were refined with anisotropic displacement parameters. The hydrogen atoms on the ligands were placed at idealized positions and refined using a riding model. We employed PLATON ${ }^{4}$ and SQUEEZE $^{5}$ to calculate the diffraction contribution of the solvent molecules and thereby produce a set of solvent-free diffraction intensities. The detailed crystallographic data and structure refinement parameters for these compounds are summarized in Table S1 (CCDC: 1960307-1960314). 


\section{Gas Sorption Measurements}

After the bulk of the solvent was decanted, the freshly prepared sample of eight mm-MOFs $(\sim 0.15 \mathrm{~g})$ was soaked in $\sim 10 \mathrm{~mL}$ of methanol for $1 \mathrm{~h}$, and then the solvent was decanted. Following the procedure of methanol soaking and decanting 10 times, the solvent-exchanged samples were activated by vacuum at $80{ }^{\circ} \mathrm{C}$ overnight $(\sim 6 \mathrm{~h})$ until a pressure of $5 \mu \mathrm{mHg}$ to obtain the activated mm-MOFs, respectively. $\mathrm{N}_{2}, \mathrm{CO}_{2}, \mathrm{C}_{2} \mathrm{H}_{2}$ adsorption isotherms were measured on Micromeritics ASAP 2020 HD88 surface area analyzer for activated mm-MOFs. As the center-controlled air condition was set up at $23{ }^{\circ} \mathrm{C}$, a water bath of $23{ }^{\circ} \mathrm{C}$ was used for adsorption isotherms at $296 \mathrm{~K}$, whereas liquid nitrogen and ice-water baths were used for the isotherms at $77 \mathrm{~K}$ and $273 \mathrm{~K}$, respectively. In order to determine the optimal activation time, $77 \mathrm{~K} \mathrm{~N}_{2}$ adsorption curves of FJU-6-TATB with different activation times were determined (Figure S11).

Adsorption Enthalpy Calculation. The $\mathrm{C}_{2} \mathrm{H}_{2}$ and $\mathrm{CO}_{2}$ adsorption enthalpy $\left(Q_{s t}\right)$ of FJU-6-TATB was calculated using adsorption data at $273 \mathrm{~K}$ and $296 \mathrm{~K}$. A virial-type expression (eq S1) was used to fit these data, and then the $\mathrm{Q}_{\text {st }}$ was then calculated by the expression given by eq $\mathrm{S} 2$.

$$
\begin{gathered}
\operatorname{InP}=\operatorname{In}(n)+\frac{1}{T} \sum_{i=0}^{m} a_{i} n^{i}+\sum_{i=0}^{n} b_{i} n^{i} \quad(\mathrm{~S} 1) \\
Q_{s t}=-R \sum_{i=0}^{m} a_{i} n^{i} \quad \text { (S2) }
\end{gathered}
$$

Here, $\mathrm{P}, \mathrm{n}$, and $\mathrm{T}$ are the pressure, amount adsorbed, and temperature, respectively. $\mathrm{m}$ and $\mathrm{n}$ determine the number of terms required to adequately describe the isotherm. $a_{i}$ and $b_{i}$ are virial coefficients.

Prediction of the Gas Adsorption Selectivity by IAST: The $\mathrm{C}_{2} \mathrm{H}_{2} / \mathrm{CO}_{2}$ selectivity of FJU-6-TATB were estimated by ideal adsorbed solution theory (IAST) theory. In this work, the measured experimental data is excess loadings $\left(\mathrm{q}^{\mathrm{ex}}\right)$ of the pure components $\mathrm{CO}_{2}$ and $\mathrm{C}_{2} \mathrm{H}_{2}$ for FJU-6-TATB, which should be converted to absolute loadings $(q)$ firstly.

$$
\mathrm{q}=q^{e x}+\frac{p V_{\text {pore }}}{Z R T}
$$

Here $\mathrm{Z}$ is the compressibility factor. The Peng-Robinson equation was used to estimate the 
value of compressibility factor to obtain the absolute loading, while the measure pore volume $0.58 \mathrm{~cm}^{3} / \mathrm{g}$ is also necessary.

The single-site Langmuir-Freundlich equation is used for fitting the isotherm data at $296 \mathrm{~K}$ and $273 \mathrm{~K}$, respectively.

$$
q=q^{\max } \frac{b p^{1 / n}}{1+b p^{1 / n}}
$$

Here $\mathrm{p}$ is the pressure of the bulk gas at equilibrium with the adsorbed phase $(\mathrm{kPa}), \mathrm{q}$ is the adsorbed amount per mass of adsorbent $(\mathrm{mmol} / \mathrm{g}), \mathrm{q}^{\max }$ is the saturation capacities of site 1 $\left(\mathrm{cm}^{3} / \mathrm{g}\right), \mathrm{b}$ is the affinity coefficients of site $1(1 / \mathrm{kPa}), \mathrm{n}$ is the deviations from an ideal homogeneous surface.

The adsorption selectivity for $\mathrm{C}_{2} \mathrm{H}_{2} / \mathrm{CO}_{2}$ separation is defined by

$$
S=\frac{q_{1} / q_{2}}{p_{1} / p_{2}}
$$

$q_{1}$ and $q_{2}$ are the molar loadings in the adsorbed phase in equilibrium with the bulk gas phase with partial pressures $p_{1}$, and $p_{2}$. We calculate the values of $q_{1}$ and $q_{2}$ using the Ideal Adsorbed Solution Theory (IAST) of Myers and Prausnitz. ${ }^{6}$

\section{Dynamic Gas Breakthrough Experiments}

The mixed-gas breakthrough separation experiment was conducted at $296 \mathrm{~K}$ using a lab scale fix-bed reactor ${ }^{7}$. In a typical experiment, $0.959 \mathrm{~g}$ of FJU-6-TATB powder was packed into a quartz tube column (the quartz tube column was $45 \mathrm{~cm}$ in length with a $5 \mathrm{~mm}$ inner diameter) with silica wool filling the void space. The sorbent was activated in-situ in the column with a vacuum pump at $353 \mathrm{~K}$ for overnight. A helium flow $(10 \mathrm{~mL} / \mathrm{min})$ was used after the activation process to purge the adsorbent. The flow of He was then turned off, while a gas mixture of $5 \% \mathrm{C}_{2} \mathrm{H}_{2}, 5 \% \mathrm{CO}_{2}$ and $90 \% \mathrm{He}$ at $3 \mathrm{~mL} / \mathrm{min}$ was allowed to flow into the column. The outlet composition was continuously monitored by gas chromatograph until complete breakthrough was achieved. On the basis of the gas balance, the gas adsorption capacities can be determined as follows ${ }^{8,9}$ :

$$
\mathrm{q}_{i}=\frac{C_{i} V}{22.4 \times m} \times \int_{0}^{t}\left(1-\frac{F}{F_{0}}\right) d t
$$


Where $\mathrm{q}_{i}$ is the equilibrium adsorption capacity of gas $i$ (mmol/g), $C_{i}$ is the feed gas concentration, $\mathrm{V}$ is the volumetric feed flow rate $\left(\mathrm{cm}^{3} / \mathrm{min}\right), \mathrm{t}$ is the adsorption time $(\mathrm{min}), \mathrm{F}_{0}$ and $\mathrm{F}$ are the inlet and outlet gas molar flow rates, respectively, and $\mathrm{m}$ is the mass of the adsorbent $(\mathrm{g})$. The separation factor $(\alpha)$ of the breakthrough experiment is determined as:

$$
\alpha=\frac{q_{1} y_{2}}{q_{2} y_{1}}
$$

In which $y_{i}$ is the molar fraction of gas $i$ in the gas mixture.

\section{Syntheses}

\section{Synthesis of pyridine-2,4,6-tribenzoic acid $\left(\mathrm{H}_{3}\right.$ PTB)}

It was synthesized according to the literature. ${ }^{10,11}$

Synthesis of FJU-6: A mixture of $\mathrm{H}_{3} \mathrm{BTB}(0.022 \mathrm{~g}, 0.05 \mathrm{mmol}), \mathrm{CoCl}_{2} \cdot 6 \mathrm{H}_{2} \mathrm{O}(0.024 \mathrm{~g}, 0.1$ mmol), HINA (0.0123 g, $0.1 \mathrm{mmol})$ and $\mathrm{H}_{2} \mathrm{BDC}(0.08 \mathrm{~g}, 0.05 \mathrm{mmol})$ were dissolved in DMA ( $2 \mathrm{~mL}$ ) by ultrasound and then added six drops of $\mathrm{HBF}_{4}(48 \%$, aq). Ultimately, the resulting blue solution was sealed in Teflon-lined stainless steel vessel $(20 \mathrm{~mL})$, which was heated in $120{ }^{\circ} \mathrm{C}$ for 2 day. After cooling to ambient temperature, pink crystals were obtained, washed by DMA and dried in air. Elemental Analysis for $\mathrm{C}_{63} \mathrm{H}_{75.5} \mathrm{~N}_{6.5} \mathrm{O}_{29} \mathrm{Co}_{4.5}=\left\{\left(\mathrm{NH}_{2}\left(\mathrm{CH}_{3}\right)_{2}\right)\left[\mathrm{Co}_{4.5}\left(\mu_{3}-\mathrm{OH}\right)\left(\mu_{4}-\mathrm{O}\right)_{0.5}(\mathrm{BTB})(\mathrm{INA})_{4}(\mathrm{BDC})_{0.5}\right](\mathrm{DMA})_{1.5}(\mathrm{H}\right.$ $\left.\left.{ }_{2} \mathrm{O}\right)_{10}\right\}_{\mathrm{n}}$ (\%) Calcd: C 45.78, H 4.60, N 6.36. Found: C 44.58, H 4.61, N 6.91.

Synthesis of FJU-6-PTB: The similar synthesis method as that for FJU-6 was applied except the ligand $\mathrm{H}_{3} \mathrm{BTB}$ was replaced by $\mathrm{H}_{3}$ PTB. Pink crystals were obtained, washed by DMA and dried in air. Elemental Analysis for $\mathrm{C}_{78} \mathrm{H}_{106.5} \mathrm{~N}_{11.5} \mathrm{O}_{31} \mathrm{Co}_{4.5}=$ $\left\{\left(\mathrm{NH}_{2}\left(\mathrm{CH}_{3}\right)_{2}\right)\left[\mathrm{Co}_{4.5}\left(\mu_{3}-\mathrm{OH}\right)\left(\mu_{4}-\mathrm{O}\right)_{0.5}(\mathrm{PTB})(\mathrm{INA})_{4}(\mathrm{BDC})_{0.5}\right](\mathrm{DMA})_{5.5}\left(\mathrm{H}_{2} \mathrm{O}\right)_{8}\right\}_{\mathrm{n}} \quad$ \% $\quad$ Calcd: C47.64, H 5.46, N 8.19. Found: C 46.55, H 5.32, N 8.12.

Synthesis of FJU-6-TATB: The similar synthesis method as that for FJU-6 was applied except the ligand $\mathrm{H}_{3}$ BTB was replaced by $\mathrm{H}_{3}$ TATB. Pink crystals were obtained, washed by DMA and dried in air. Elemental Analysis for $\mathrm{C}_{62} \mathrm{H}_{87} \mathrm{~N}_{10} \mathrm{O}_{34.5} \mathrm{Co}_{4.5}=$ 
$\left\{\left(\mathrm{NH}_{2}\left(\mathrm{CH}_{3}\right)_{2}\right)\left[\mathrm{Co}_{4.5}\left(\mu_{3}-\mathrm{OH}\right)\left(\mu_{4}-\mathrm{O}\right)_{0.5}(\mathrm{TATB})(\mathrm{INA})_{4}(\mathrm{BDC})_{0.5}\right](\mathrm{DMA})_{2}\left(\mathrm{H}_{2} \mathrm{O}\right)_{15}\right\}_{\mathrm{n}} \quad$ (\%) Calcd: C 41.61, H 4.90, N 7.83. Found: C 40.02, H 4.81, N 7.62.

Synthesis of FJU-6-Me-INA: The similar synthesis method as that for FJU-6 was applied except the ligand HINA was replaced by Me-HINA. Pink crystals were obtained, washed by DMA and dried in air. Elemental Analysis for $\mathrm{C}_{71} \mathrm{H}_{84.5} \mathrm{~N}_{7.5} \mathrm{O}_{26} \mathrm{Co}_{4.5}=$ $\left\{\left(\mathrm{NH}_{2}\left(\mathrm{CH}_{3}\right)_{2}\right)\left[\mathrm{Co}_{4.5}\left(\mu_{3}-\mathrm{OH}\right)\left(\mu_{4}-\mathrm{O}\right)_{0.5}(\mathrm{BTB})(\mathrm{Me}-\mathrm{INA})_{4}(\mathrm{BDC})_{0.5}\right](\mathrm{DMA})_{2.5}\left(\mathrm{H}_{2} \mathrm{O}\right)_{6}\right\}_{\mathrm{n}}(\%)$ Calcd: C 49.46, H 4.94, N 6.09. Found: C 49.32, H 5.07, N 5.84.

Synthesis of FJU-6-F-INA: The similar synthesis method as that for FJU-6 was applied except the ligand HINA was replaced by F-HINA. Pink crystals were obtained, washed by $D M A$ and dried in air. Elemental Analysis for $\mathrm{C}_{89} \mathrm{H}_{112} \mathrm{~N}_{13} \mathrm{O}_{26.5} \mathrm{~F}_{4} \mathrm{Co}_{4.5}=$ $\left\{\left(\mathrm{NH}_{2}\left(\mathrm{CH}_{3}\right)_{2}\right)\left[\mathrm{Co}_{4.5}\left(\mu_{3}-\mathrm{OH}\right)\left(\mu_{4}-\mathrm{O}\right)_{0.5}(\mathrm{BTB})(\mathrm{F}-\mathrm{INA})_{4}(\mathrm{BDC})_{0.5}\right](\mathrm{DMA})_{8}\left(\mathrm{H}_{2} \mathrm{O}\right)\right\}_{\mathrm{n}} \quad(\%)$ Calcd: C 50.21, H 5.30, N 8.55. Found: C 50.59, H 5.25, N 8.88.

Synthesis of FJU-6-NDC: The similar synthesis method as that for FJU-6 was applied except the ligand $\mathrm{H}_{2} \mathrm{BDC}$ was replaced by $\mathrm{H}_{2} \mathrm{NDC}$. Pink crystals were obtained, washed by DMA and dried in air. Elemental Analysis for $\mathrm{C}_{77} \mathrm{H}_{93.5} \mathrm{~N}_{9.5} \mathrm{O}_{27} \mathrm{Co}_{4.5}=$ $\left\{\left(\mathrm{NH}_{2}\left(\mathrm{CH}_{3}\right)_{2}\right)\left[\mathrm{Co}_{4.5}\left(\mu_{3}-\mathrm{OH}\right)\left(\mu_{4}-\mathrm{O}\right)_{0.5}(\mathrm{BTB})(\mathrm{INA})_{4}(\mathrm{NDC})_{0.5}\right](\mathrm{DMA})_{4.5}\left(\mathrm{H}_{2} \mathrm{O}\right)_{5}\right\}_{\mathrm{n}} \quad(\%)$ Calcd: C 50.01, H 5.09, N 7.20. Found: C 49.85, H 4.95, N 7.16.

Synthesis of FJU-6-BrBDC: The similar synthesis method as that for FJU-6 was applied except the ligand $\mathrm{H}_{2} \mathrm{BDC}$ was replaced by $\mathrm{Br}-\mathrm{H}_{2} \mathrm{BDC}$. Pink crystals were obtained, washed by DMA and dried in air. Elemental Analysis for $\mathrm{C}_{81} \mathrm{H}_{105.5} \mathrm{~N}_{11} \mathrm{O}_{28.5} \mathrm{Co}_{4.5} \mathrm{Br}_{0.5}=$ $\left\{\left(\mathrm{NH}_{2}\left(\mathrm{CH}_{3}\right)_{2}\right)\left[\mathrm{Co}_{4.5}\left(\mu_{3}-\mathrm{OH}\right)\left(\mu_{4}-\mathrm{O}\right)_{0.5}(\mathrm{BTB})(\mathrm{INA})_{4}(\mathrm{BrBDC})_{0.5}\right](\mathrm{DMA})_{6}\left(\mathrm{H}_{2} \mathrm{O}\right)_{5}\right\}_{\mathrm{n}} \quad(\%)$ Calcd: C 48.78, H 5.33, N 7.72. Found: C 48.74, H 5.39, N 7.89.

Synthesis of FJU-6-Ni: The similar synthesis method as that for FJU-6 was applied except the metal $\mathrm{CoCl}_{2} \cdot 6 \mathrm{H}_{2} \mathrm{O}$ was replaced by $\mathrm{NiCl}_{2} \cdot 6 \mathrm{H}_{2} \mathrm{O}$. Grass green crystals were obtained, washed by DMA and dried in air. Elemental Analysis for $\mathrm{C}_{73} \mathrm{H}_{88} \mathrm{~N}_{9} \mathrm{O}_{26.5} \mathrm{Ni}_{4.5}=$ 
$\left\{\left(\mathrm{NH}_{2}\left(\mathrm{CH}_{3}\right)_{2}\right)\left[\mathrm{Ni}_{4.5}\left(\mu_{3}-\mathrm{OH}\right)\left(\mu_{4}-\mathrm{O}\right)_{0.5}(\mathrm{BTB})(\mathrm{INA})_{4}(\mathrm{BDC})_{0.5}\right](\mathrm{DMA})_{4}\left(\mathrm{H}_{2} \mathrm{O}\right)_{5}\right\}_{\mathrm{n}} \quad(\%) \quad$ Calcd: $\quad \mathrm{C}$ 49.27, H 4.98, N 7.08. Found: C 48.10, H 4.92, N 7.09. 
Table S1 X-ray crystallography: summary of data collection and refinement details.

\begin{tabular}{|c|c|c|c|c|c|c|c|c|}
\hline MOF & FJU-6 & FJU-6-PTB & FJU-6-TATB & FJU-6-Me-INA & FJU-6-F-INA & FJU-6-NDC & FJU-6-BrBDC & FJU-6-Ni \\
\hline Formula & $\mathrm{C}_{55} \mathrm{H}_{33} \mathrm{Co}_{4.5} \mathrm{~N}_{4} \mathrm{O}_{17.5}$ & $\mathrm{C}_{54} \mathrm{H}_{32} \mathrm{Co}_{4.5} \mathrm{~N}_{5} \mathrm{O}_{17.5}$ & $\mathrm{C}_{52} \mathrm{H}_{30} \mathrm{Co}_{4.5} \mathrm{~N}_{7} \mathrm{O}_{17.5}$ & $\mathrm{C}_{58} \mathrm{H}_{40} \mathrm{Co}_{4.5} \mathrm{~N}_{4} \mathrm{O}_{17.5}$ & $\mathrm{C}_{55} \mathrm{H}_{23.5} \mathrm{Co}_{4.5} \mathrm{~F}_{4} \mathrm{~N}_{4} \mathrm{O}_{17.5}$ & $\mathrm{C}_{57} \mathrm{H}_{33} \mathrm{Co}_{4.5} \mathrm{~N}_{4} \mathrm{O}_{17.5}$ & $\mathrm{C}_{55} \mathrm{H}_{31} \mathrm{Br}_{0.5} \mathrm{Co}_{4.5} \mathrm{~N}_{4} \mathrm{O}_{17.5}$ & $\mathrm{C}_{55} \mathrm{H}_{34} \mathrm{~N}_{4} \mathrm{Ni}_{4.5} \mathrm{O}_{17.5}$ \\
\hline Formula weight & 1295.04 & 1296.03 & 1298.01 & 1338.12 & 1361.46 & 1319.06 & 1332.98 & 1295.05 \\
\hline Temperature/K & 291.10(10) & 273.15 & $294.08(17)$ & $293.6(4)$ & $100.00(10)$ & $293.26(16)$ & 294.1(6) & $292.17(10)$ \\
\hline Crystal system & tetragonal & tetragonal & tetragonal & tetragonal & tetragonal & tetragonal & tetragonal & tetragonal \\
\hline Space group & $P \overline{4 n} 2$ & $P \overline{4 n} 2$ & $P \overline{4 n} 2$ & $P \overline{4 n} 2$ & $P \overline{4 n} 2$ & $P \overline{4 n} 2$ & $P \overline{4 n} 2$ & $P \overline{4 n} 2$ \\
\hline $\mathrm{a} / \AA$ & $21.8423(5)$ & $21.81320(10)$ & $21.7735(5)$ & $21.8598(3)$ & $21.8545(3)$ & $21.9069(4)$ & 21.9081(4) & $21.6973(5)$ \\
\hline $\mathrm{b} / \AA$ & $21.8423(5)$ & $21.81320(10)$ & $21.7735(5)$ & $21.8598(3)$ & $21.8545(3)$ & $21.9069(4)$ & $21.9081(4)$ & $21.6973(5)$ \\
\hline $\mathrm{c} / \AA$ & $39.3731(18)$ & $39.0403(9)$ & $39.2031(16)$ & $39.3559(11)$ & $39.3775(9)$ & $39.6456(13)$ & $39.4769(13)$ & $39.0746(19)$ \\
\hline$\alpha /^{\circ}$ & 90 & 90 & 90 & 90 & 90 & 90 & 90 & 90 \\
\hline$\beta /{ }^{\circ}$ & 90 & 90 & 90 & 90 & 90 & 90 & 90 & 90 \\
\hline$\gamma /{ }^{\circ}$ & 90 & 90 & 90 & 90 & 90 & 90 & 90 & 90 \\
\hline Volume $/ \AA^{3}$ & $18784.3(12)$ & $18576.0(5)$ & $18585.7(11)$ & $18806.2(7)$ & $18807.4(7)$ & 19026.3(10) & 18947.5(9) & 18395.3(12) \\
\hline $\mathrm{Z}$ & 8 & 8 & 8 & 8 & 8 & 8 & 8 & 8 \\
\hline$\rho_{\text {calc }} \mathrm{g} / \mathrm{cm}^{3}$ & 0.916 & 0.927 & 0.928 & 0.945 & 0.962 & 0.921 & 0.935 & 0.935 \\
\hline Radiation & $\mathrm{MoK} \alpha$ & $\operatorname{MoK} \alpha$ & $\operatorname{MoK} \alpha$ & $\mathrm{CuK} \alpha$ & $\mathrm{CuK} \alpha$ & $\operatorname{MoK} \alpha$ & $\mathrm{CuK} \alpha$ & $\mathrm{CuK} \alpha$ \\
\hline $\mathrm{R}$ indexes for & $R_{I}^{\mathrm{a}}=0.0969$ & $R_{I}^{\mathrm{a}}=0.0883$ & $R_{I}^{a}=0.0917$ & $R_{I}^{a}=0.1169$ & $R_{I}^{a}=0.1049$ & $R_{I}^{a}=0.0922$ & $R_{l}^{a}=0.1271$ & $R_{l}^{a}=0.0914$ \\
\hline $\mathrm{I}>=2 \sigma(\mathrm{I})$ & $w R_{2}^{\mathrm{b}}=0.2280$ & $w R_{2}^{\mathrm{b}}=0.2598$ & $w R_{2}^{b}=0.2423$ & $w R_{2}^{b}=0.3007$ & $w R_{2}^{b}=0.2766$ & $w R_{2}^{b}=0.2674$ & $w R_{2}^{b}=0.3118$ & $w R_{2}^{b}=0.2240$ \\
\hline $\mathrm{R}$ indexes for & $R_{l}^{\mathrm{a}}=0.1809$ & $R_{I}^{\mathrm{a}}=0.1055$ & $R_{l}^{a}=0.1557$ & $R_{1}^{a}=0.1755$ & $R_{1}^{a}=0.1564$ & $R_{I}^{a}=0.1149$ & $R_{I}^{a}=0.2274$ & $R_{l}^{a}=0.1852$ \\
\hline all data & $w R_{2}^{\mathrm{b}}=0.2788$ & $w R_{2}^{\mathrm{b}}=0.2815$ & $w R_{2}^{b}=0.2796$ & $w R_{2}^{b}=0.3547$ & $w R_{2}^{b}=0.3256$ & $w R_{2}^{b}=0.2939$ & $w R_{2}^{b}=0.4037$ & $w R_{2}^{b}=0.2853$ \\
\hline
\end{tabular}

${ }^{\mathrm{a}} \mathrm{R}_{1}=\sum\left(\left|\mathrm{F}_{\mathrm{o}}\right|-\left|\mathrm{F}_{\mathrm{c}}\right|\right) / \sum\left|\mathrm{F}_{\mathrm{o}}\right|,{ }^{\mathrm{b}} \mathrm{wR}_{2}=\left[\sum \mathrm{w}\left(\mathrm{F}_{\mathrm{o}}{ }^{2}-\mathrm{F}_{\mathrm{c}}{ }^{2}\right)^{2} / \sum \mathrm{w}\left(\mathrm{F}_{\mathrm{o}}{ }^{2}\right)^{2}\right]^{0.5}$ 
Table S2 BVS analyses of $\mathrm{Co}, \mu_{3}-\mathrm{O}$ and $\mu_{4}-\mathrm{O}$ atoms for FJU-6

\begin{tabular}{|c|c|c|c|c|c|}
\hline & Co1 & & & $\mathrm{Co} 2$ & \\
\hline bond & $d_{i j}(\AA)$ & $V_{i j}$ & bond & $d_{i j}(\AA)$ & $V_{i j}$ \\
\hline Co1-O8 & 2.076 & 0.354 & Co2-O9 & 2.121 & 0.314 \\
\hline Co1-O12 & 2.046 & 0.384 & $\mathrm{Co} 2-\mathrm{O} 7$ & 2.08 & 0.350 \\
\hline Co1-O1 & 2.175 & 0.271 & $\mathrm{Co} 2-\mathrm{O} 4$ & 2.085 & 0.346 \\
\hline Co1-N4 & 2.223 & 0.356 & Co2-O6 & 2.045 & 0.385 \\
\hline Co1-O18 & 2.048 & 0.382 & Co2-O18 & 2.013 & 0.420 \\
\hline \multirow[t]{3}{*}{ Co1-O10 } & 2.111 & 0.322 & Co2-N2 & 2.083 & 0.519 \\
\hline & $V_{i}$ & 2.069 & & $V_{i}$ & 2.334 \\
\hline & $\mathrm{Co} 3$ & & & $\mathrm{Co} 4$ & \\
\hline bond & $d_{i j}(\AA)$ & $V_{i j}$ & bond & $d_{i j}(\AA)$ & $V_{i j}$ \\
\hline $\mathrm{Co} 3-\mathrm{O} 2$ & 2.072 & 0.358 & Co4-O13 & 2.053 & 0.377 \\
\hline Co2-O11 & 2.114 & 0.320 & Co4-O17 & 2.086 & 0.344 \\
\hline $\mathrm{Co} 3-\mathrm{O} 3$ & 2.035 & 0.396 & Co4-O17 & 2.087 & 0.344 \\
\hline $\mathrm{Co} 3-\mathrm{O} 5$ & 1.994 & 0.442 & $\mathrm{Co} 4-\mathrm{O} 16$ & 2.114 & 0.320 \\
\hline $\mathrm{Co} 3-\mathrm{O} 18$ & 2.016 & 0.417 & Co4-O13 & 2.053 & 0.377 \\
\hline \multirow[t]{3}{*}{ Co3-N1 } & 2.158 & 0.423 & Co4-O16 & 2.115 & 0.319 \\
\hline & $V_{i}$ & 2.356 & & $V_{i}$ & 2.081 \\
\hline & $\mathrm{Co} 5$ & & & $\mu_{3}-\mathrm{O} 18$ & \\
\hline bond & $d_{i j}(\AA)$ & $V_{i j}$ & bond & $d_{i j}(\AA)$ & $V_{i j}$ \\
\hline Co5-N3 & 2.138 & 0.447 & O18-Co1 & 2.048 & 0.382 \\
\hline Co5-O15 & 2.129 & 0.307 & $\mathrm{O} 18-\mathrm{Co} 2$ & 2.013 & 0.420 \\
\hline Co5-O14 & 2.13 & 0.306 & O18-Co3 & 2.016 & 0.417 \\
\hline Co5-O17 & 2.066 & 0.364 & & $V_{i}$ & 1.219 \\
\hline \multirow[t]{7}{*}{ Co5-O15 } & 2.325 & 0.181 & & $\mu_{4}-\mathrm{O} 17$ & \\
\hline & $V_{i}$ & 1.605 & bond & $d_{i j}(\AA)$ & $V_{i j}$ \\
\hline & & & O17-Co5 & 2.066 & 0.364 \\
\hline & & & O17-Co5 & 2.066 & 0.364 \\
\hline & & & O17-Co4 & 2.086 & 0.345 \\
\hline & & & O17-Co4 & 2.087 & 0.344 \\
\hline & & & & $V_{i}$ & 1.417 \\
\hline
\end{tabular}

Here the valence $V_{i j}=e^{\frac{R_{0}-d_{i j}}{B}}$ and $V_{i}= \pm \sum_{j} V_{i j} \cdot \mathrm{d}_{i j}$ is the bond length between the two ions. $R_{0}$ is the reference bond length with $1.692 \AA$ for $\mathrm{Co}-\mathrm{O}$ bond and $1.84 \AA$ for $\mathrm{Co}-\mathrm{N}$ bond. $B$ is a constant approximately equal to $0.37 \AA$. 
(a)

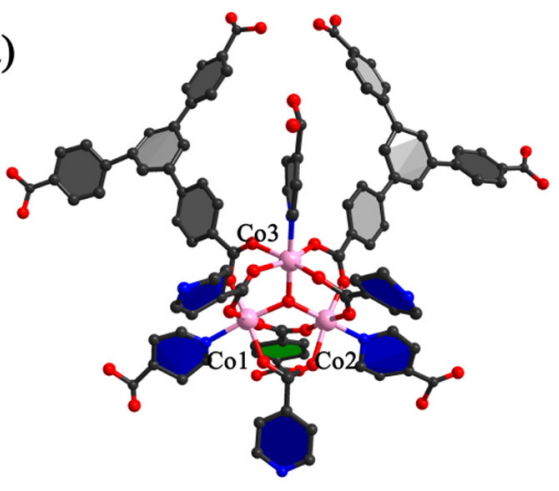

(b)

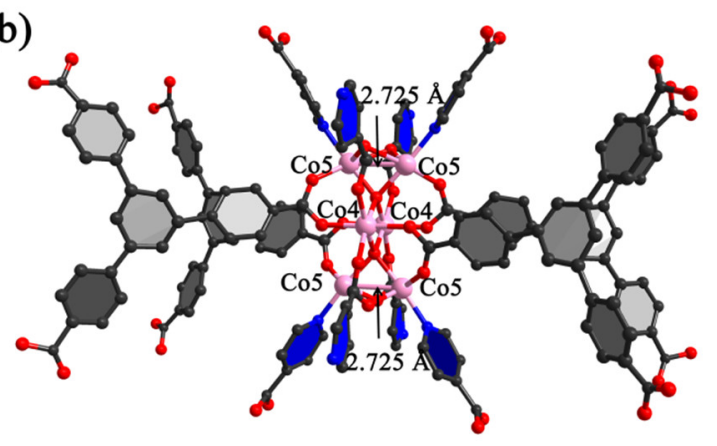

Figure S1 Coordination environments of trinuclear $\left[\mathrm{Co}_{3}\left(\mu_{3}-\mathrm{OH}\right) \mathrm{N}_{3}(\mathrm{COO})_{6}\right]$ cluster and hexanuclear $\left[\mathrm{Co}_{6}\left(\mu_{4}-\mathrm{O}\right)_{2} \mathrm{~N}_{4}(\mathrm{COO})_{8}\right]$ cluster in FJU-6.
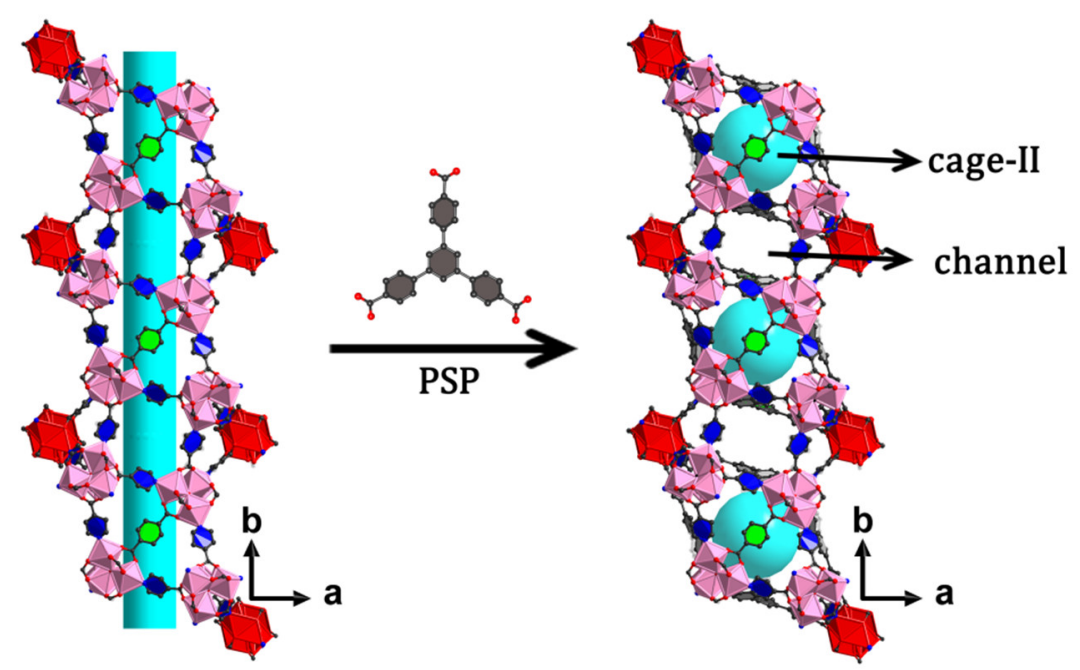

Figure S2 Pore space partition through symmetry-matching regulated ligand BTB insertion, which form cage-II and square channels in FJU-6 along the c axis.

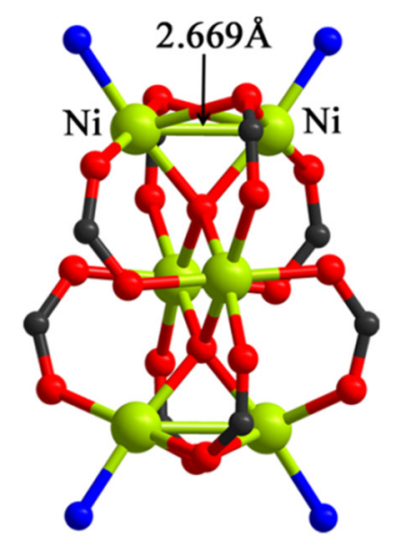

Figure $\mathbf{S 3}$ The hexanuclear $\left[\mathrm{Ni}_{6}\left(\mu_{4}-\mathrm{O}\right)_{2} \mathrm{~N}_{4}(\mathrm{COO})_{8}\right]$ cluster in $\mathbf{F J U}-6-\mathbf{N i}$. 

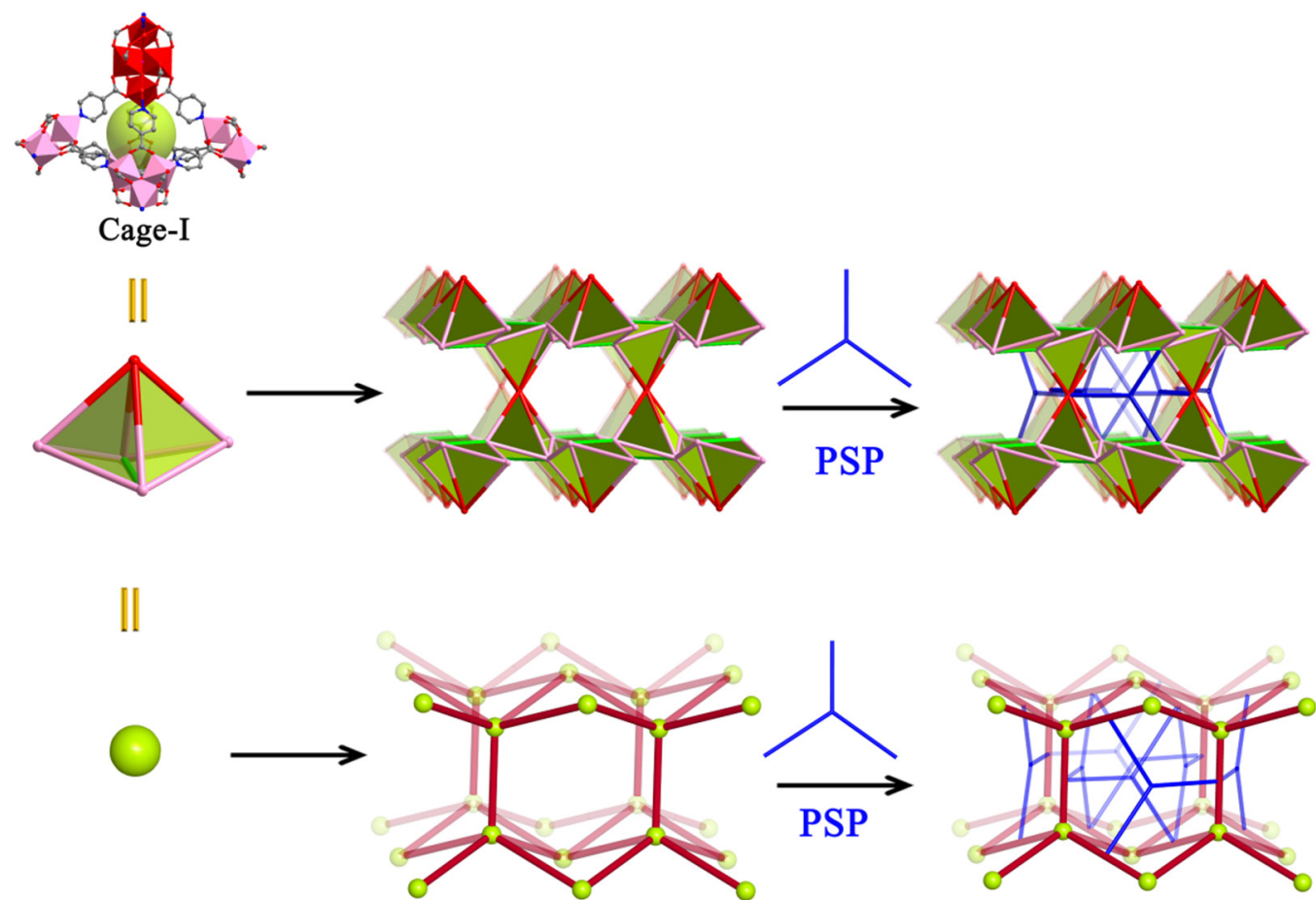

sqp net

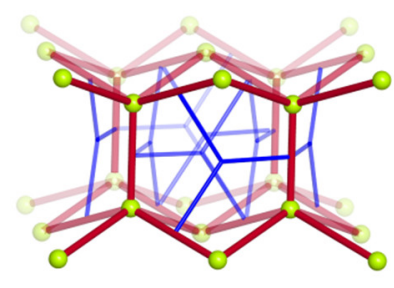

$11 z$ net

Figure S4 Taking cage as node, $l l z$ net can be obtained by modifying sqp net with PSP. 
(a)

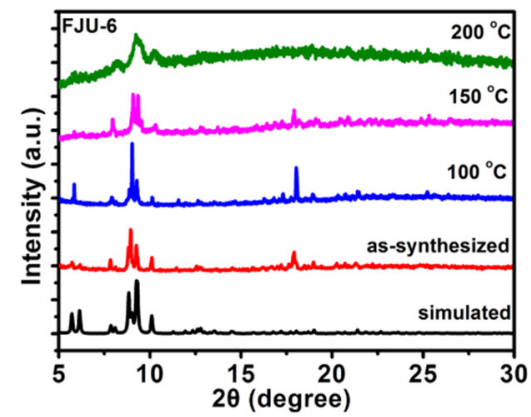

(c)

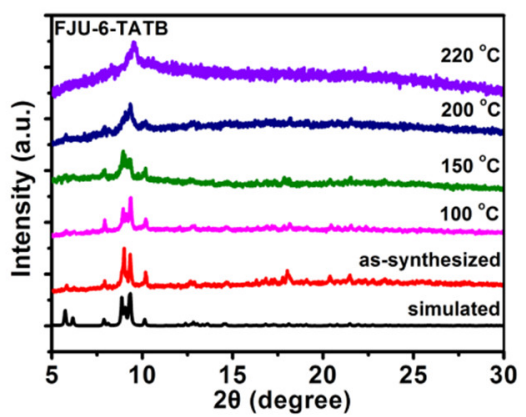

(e)

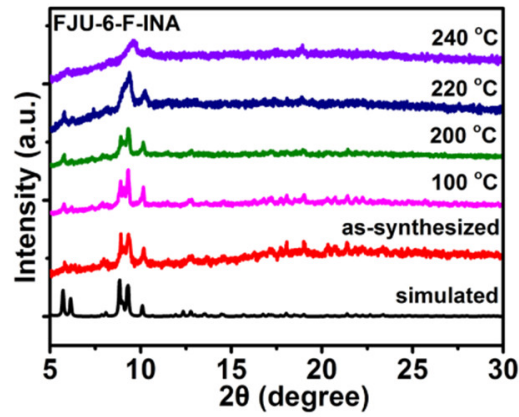

(g)

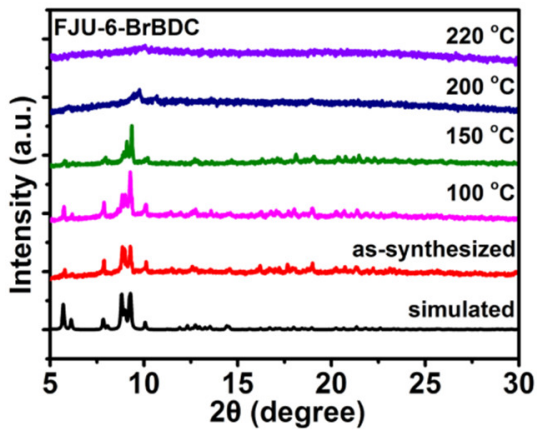

(b)

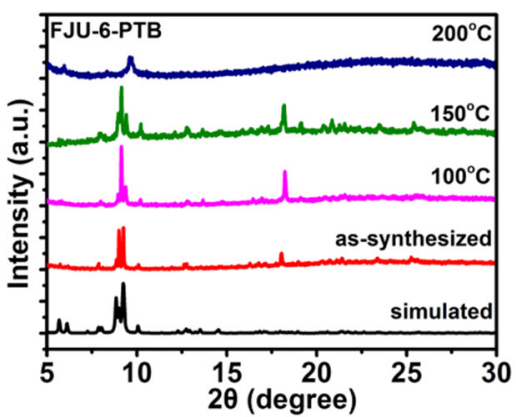

(d)

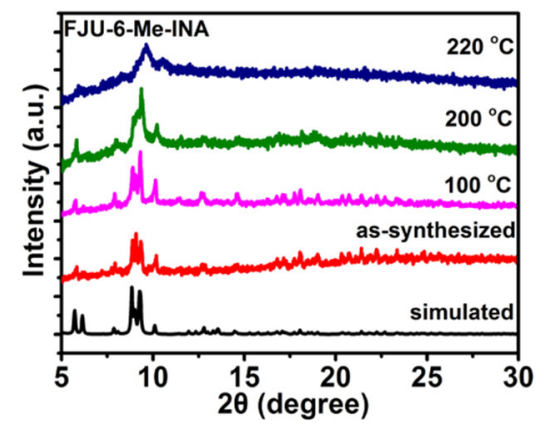

(f)

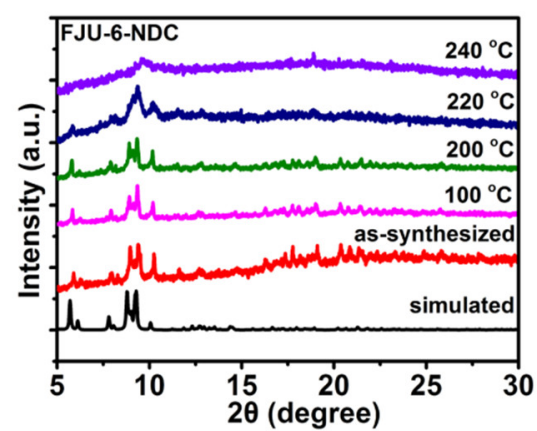

(h)

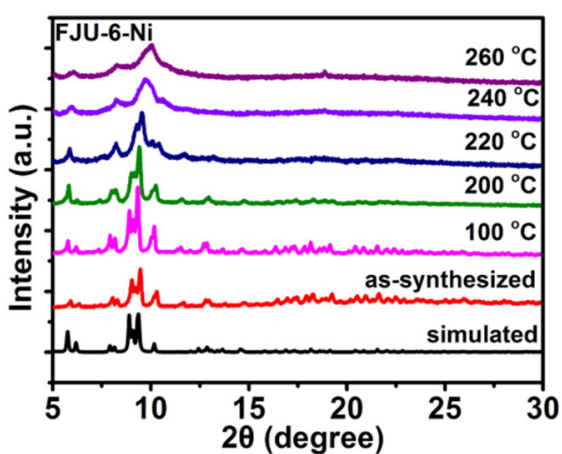

Figure S5 PXRD patterns of eight mm-MOFs after heated at different temperatures. 
(a)

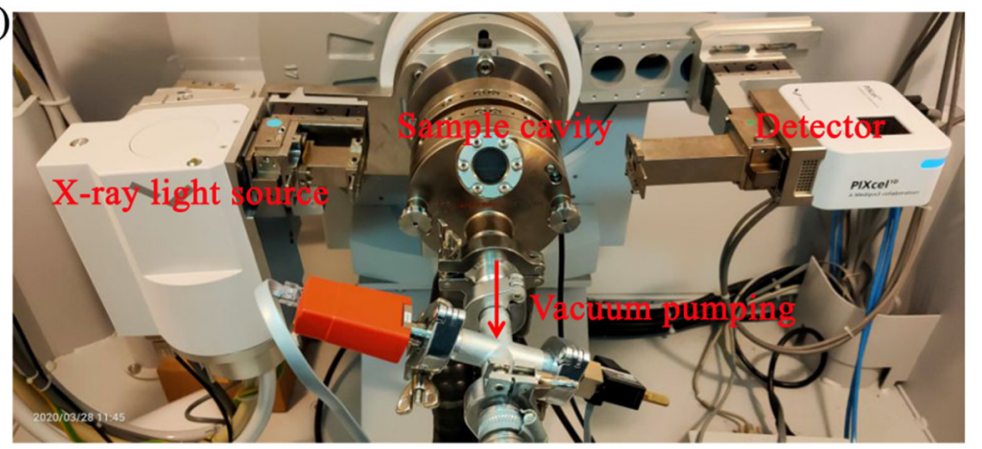

(b)

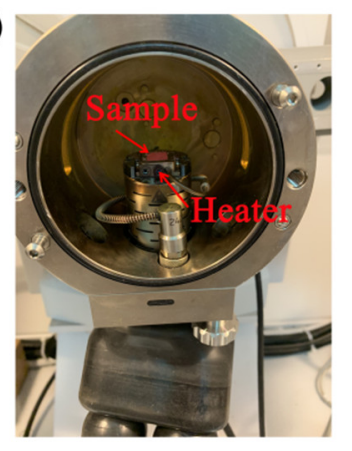

Figure S6 (a) Illustration of the in-situ activated PXRD device; (b) Internal structure of sample cavity. 
(a)

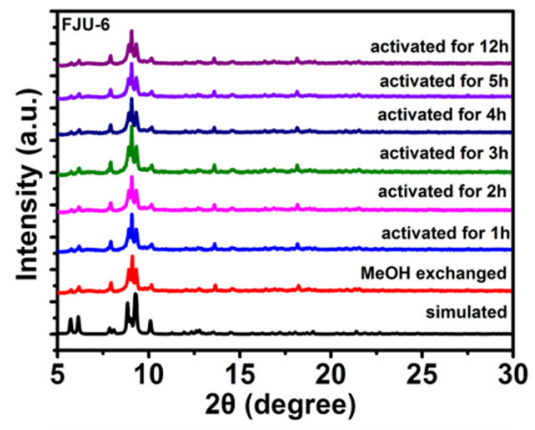

(c)

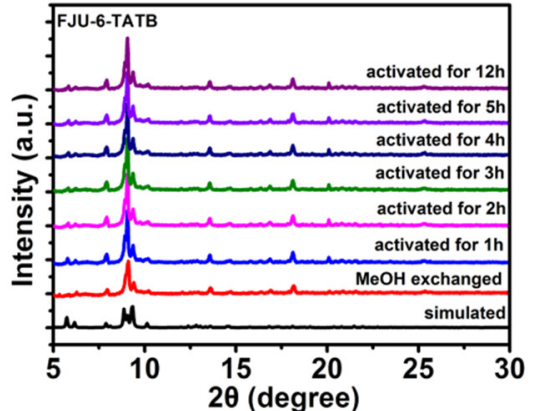

(e)

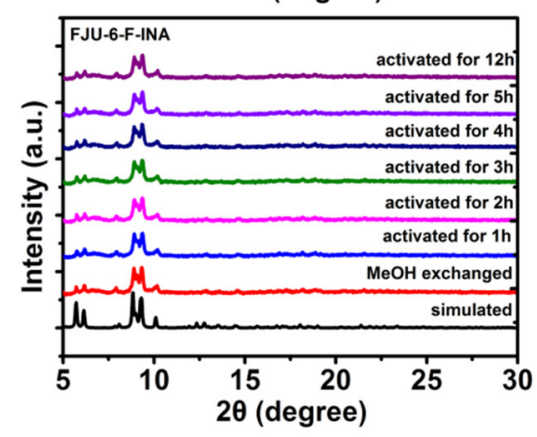

(g)

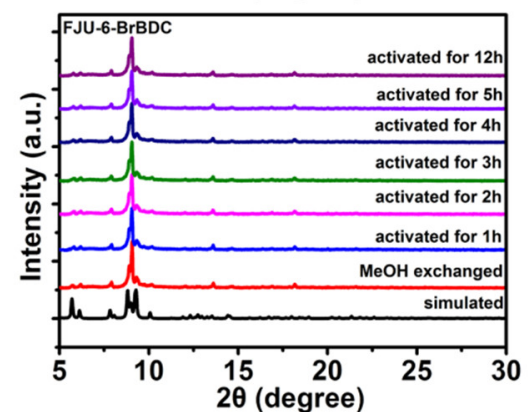

(b)

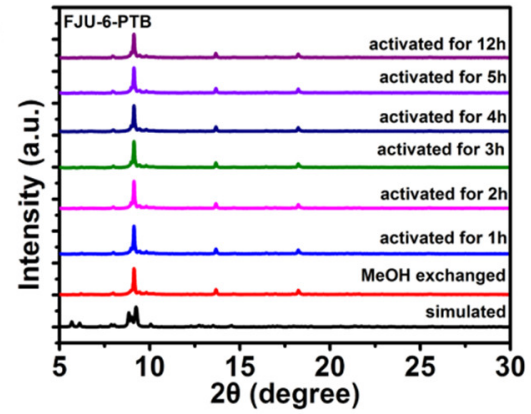

(d)

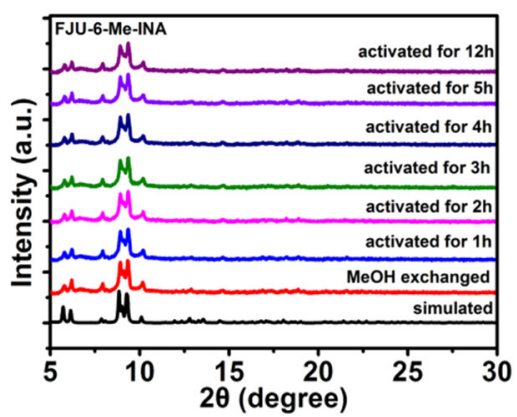

(f)

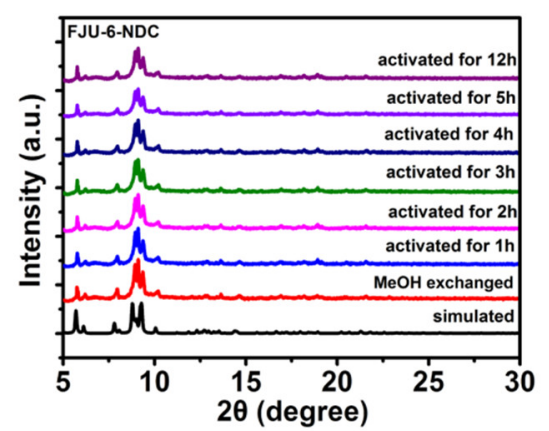

(h)

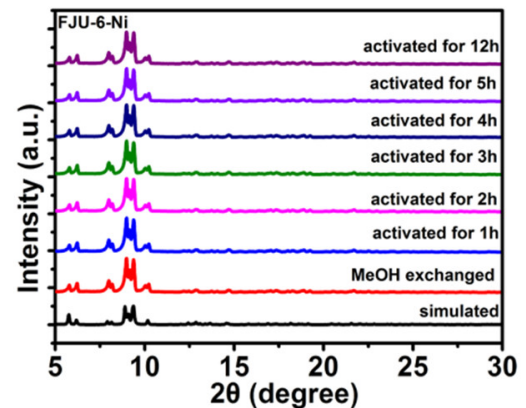

Figure S7 PXRD patterns of eight mm-MOFs after vacuum in situ activation for $12 \mathrm{~h}$. 


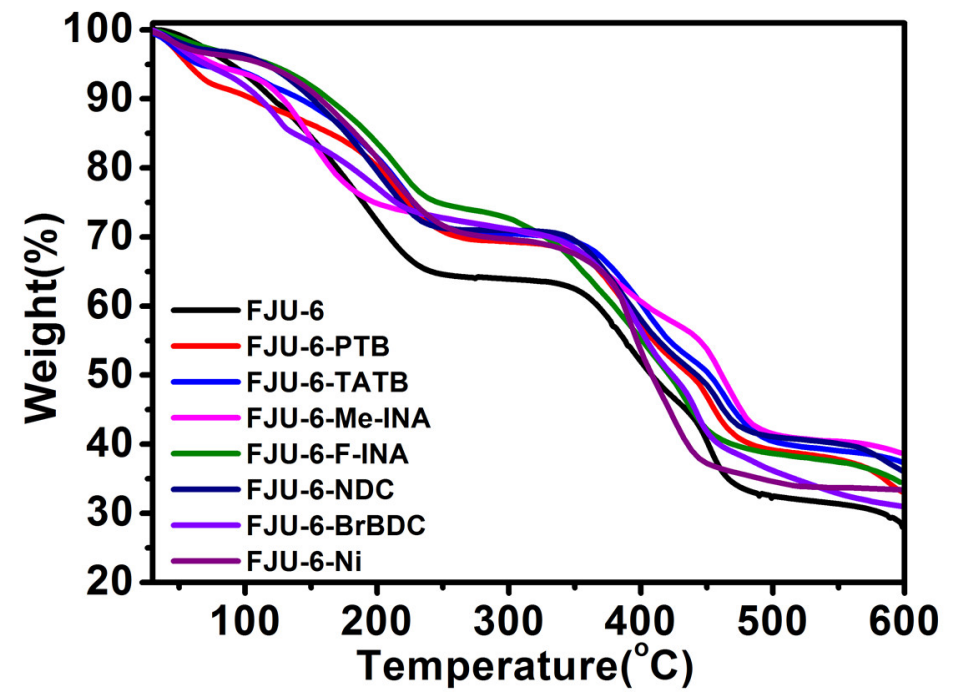

Figure S8 TGA curve of eight mm-MOFs. 


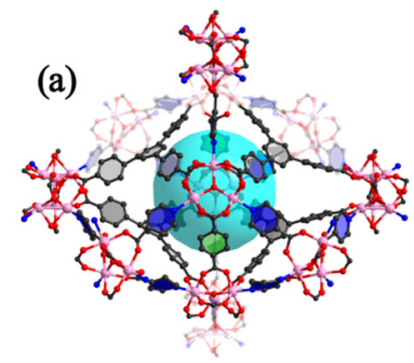

FJU-6

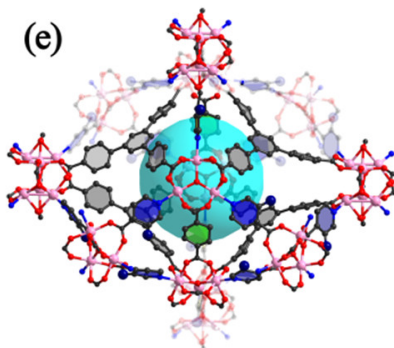

FJU-6-F-INA (b)

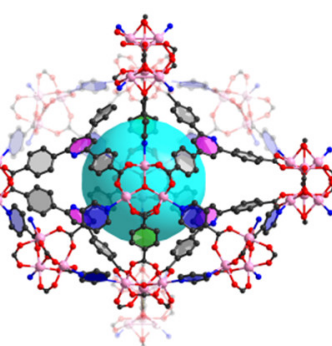

FJU-6-PTB (c)

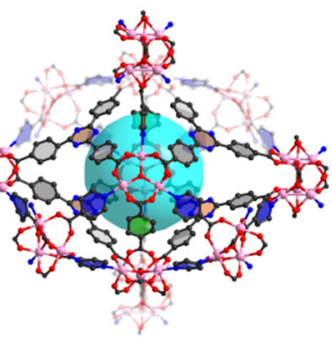

FJU-6-TATB (d)
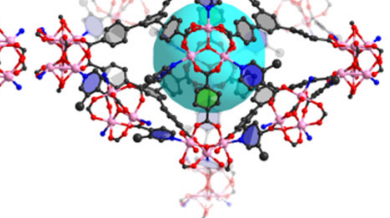

FJU-6-Me-INA (f) (g)

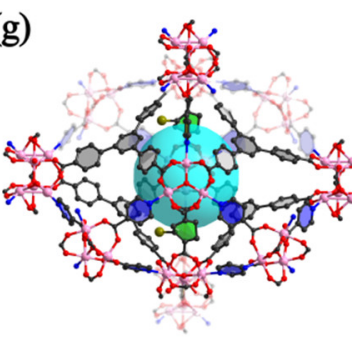

FJU-6-BrBDC (h)

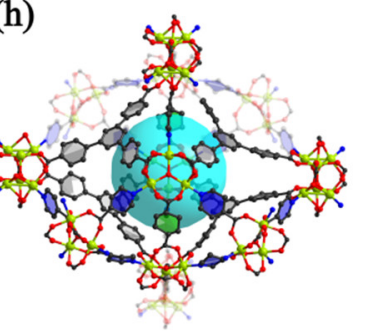

FJU-6-Ni

Figure S9 Systematic modulation of the ligands and metals of FJU-6 to produce mm-MOFs. 


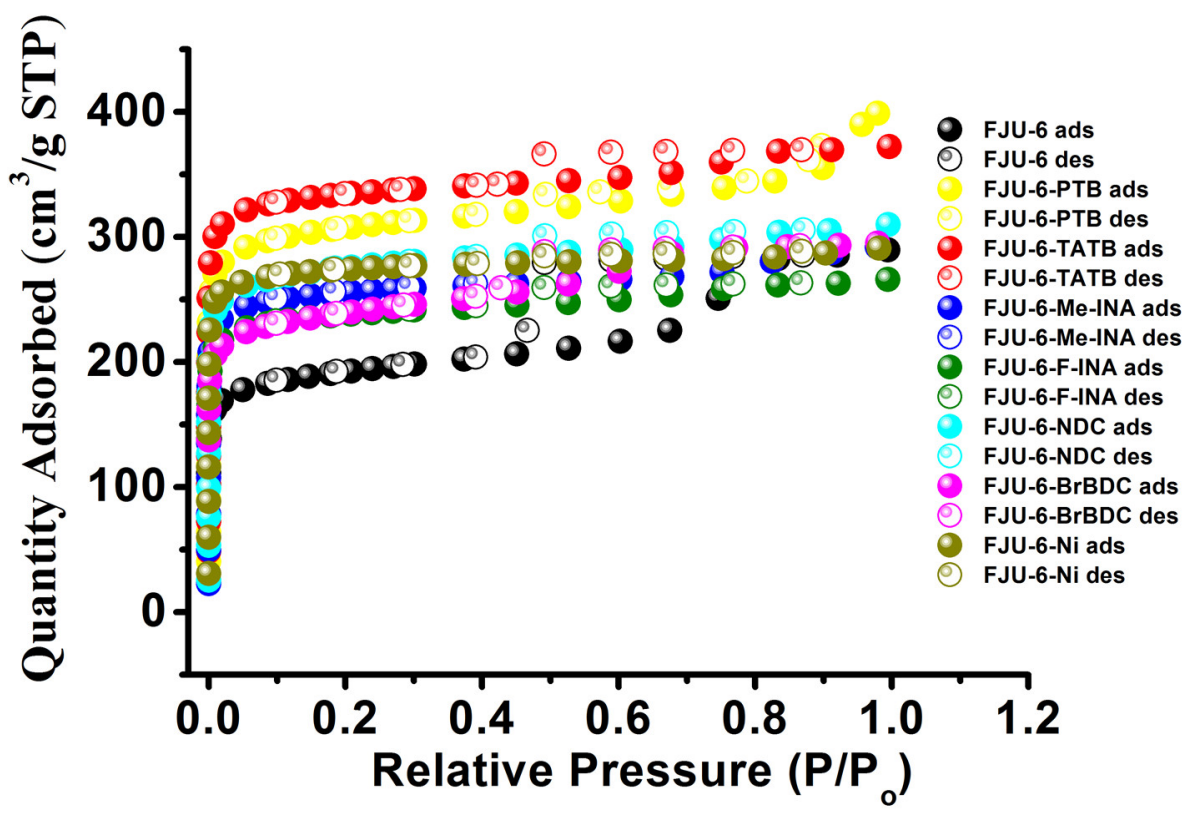

Figure S10 $77 \mathrm{~K} \mathrm{~N}_{2}$ isotherms for eight mm-MOFs.

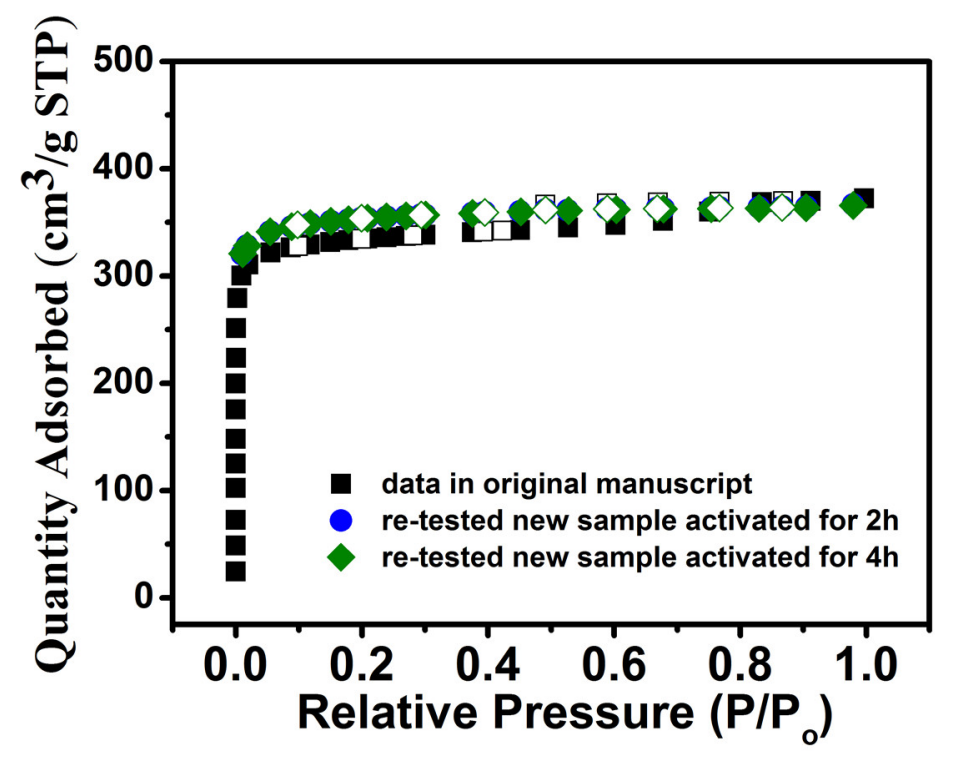

Figure S11 $77 \mathrm{~K} \mathrm{~N}_{2}$ isotherms of FJU-6-TATB at different activation times indicate that the framework is stable after activation. 


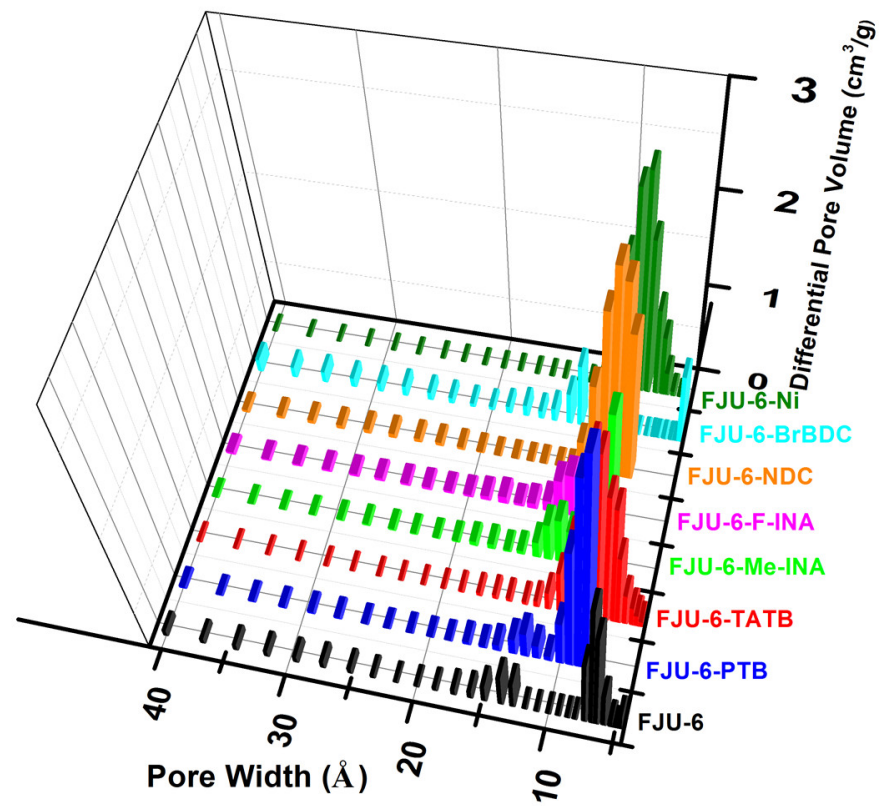

Figure S12. Pore size distribution plots for eight mm-MOFs, calculated using a DFT method from $77 \mathrm{~K} \mathrm{~N}_{2}$ isotherms.

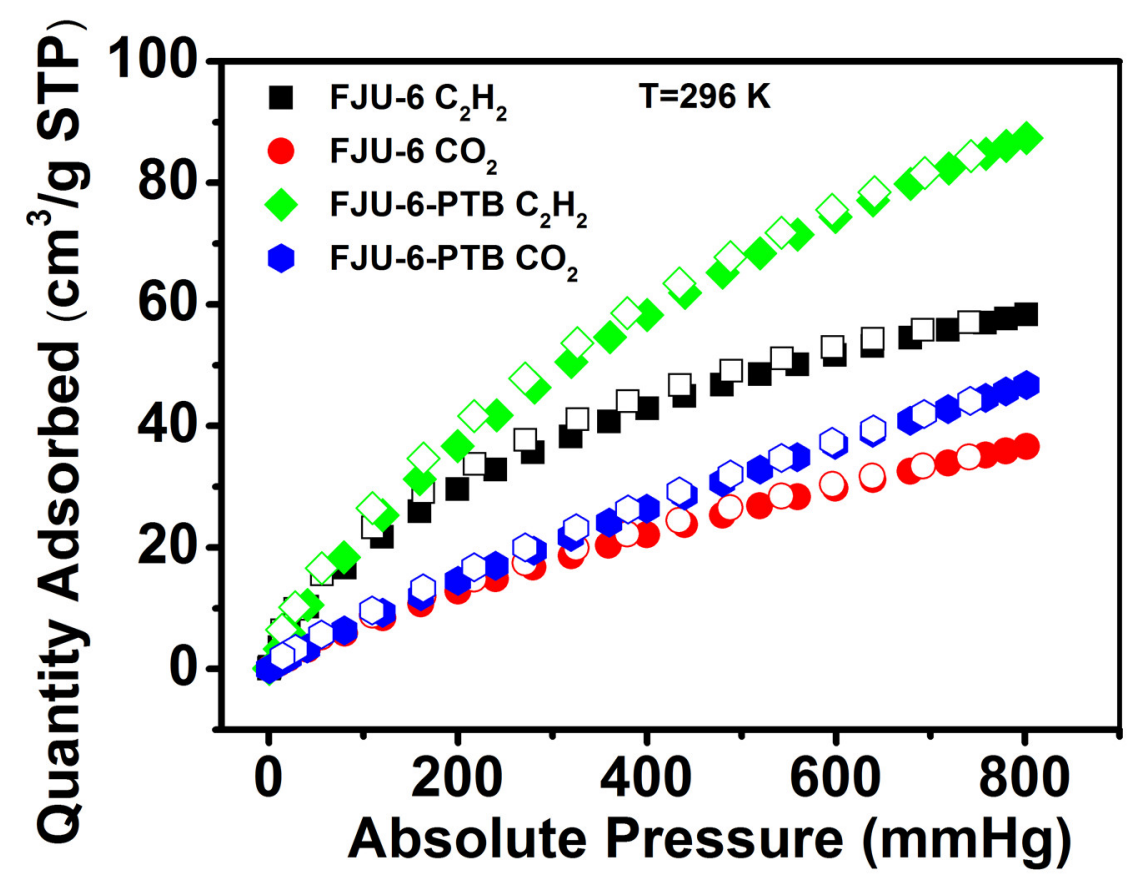

Figure S13. Adsorption isotherms for $\mathrm{C}_{2} \mathrm{H}_{2}$ and $\mathrm{CO}_{2}$ of FJU-6 and FJU-6-PTB, at $296 \mathrm{~K}$. 


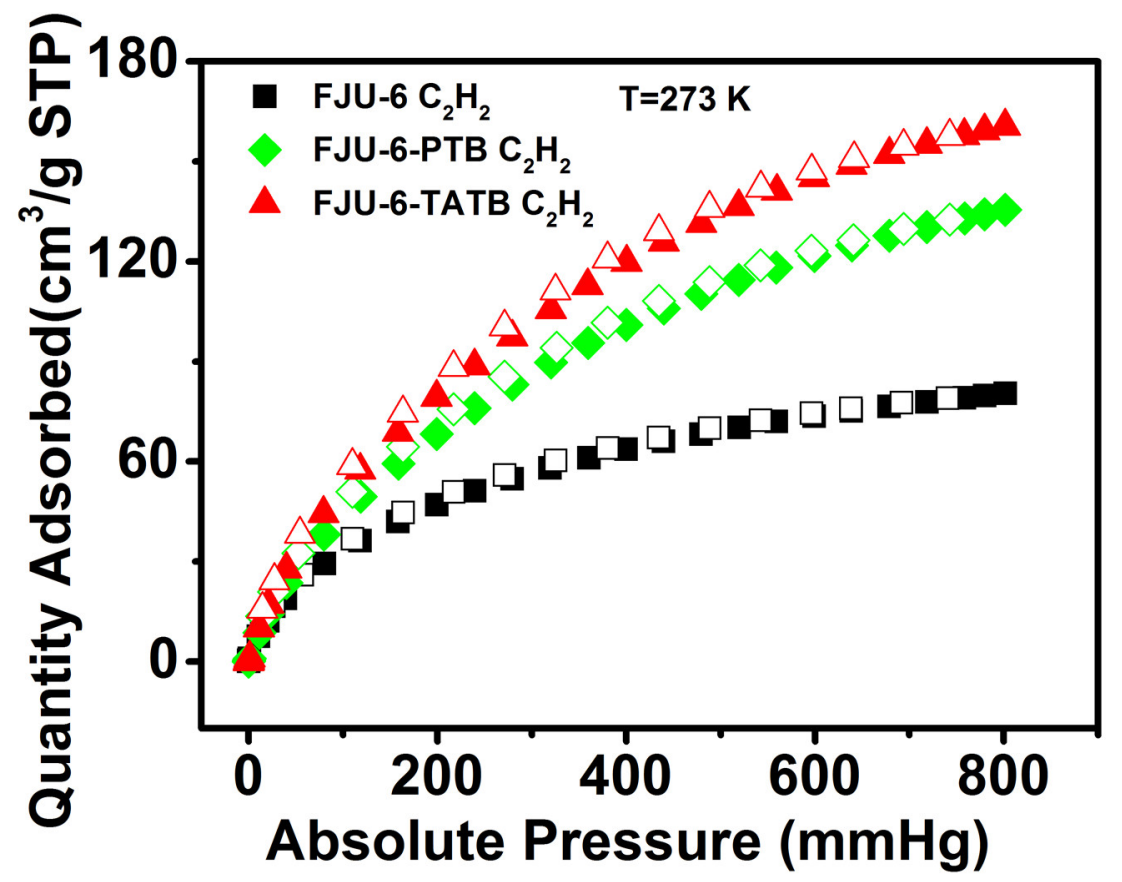

Figure S14. Adsorption isotherms for $\mathrm{C}_{2} \mathrm{H}_{2}$ of FJU-6, FJU-6-PTB, and FJU-6-TATB at $273 \mathrm{~K}$.
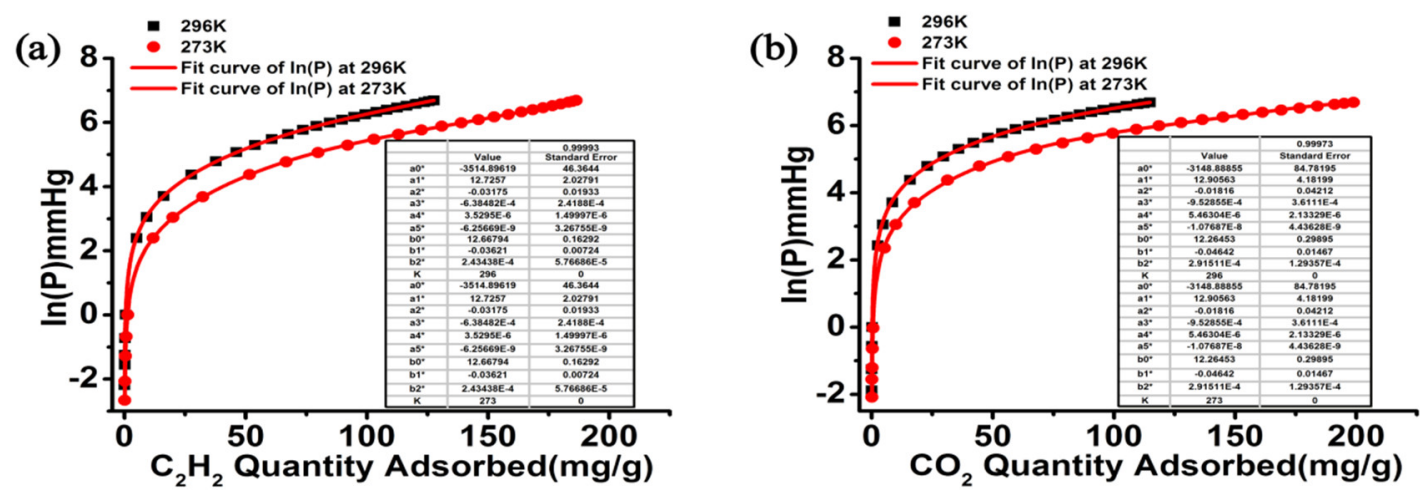

Figure S15. The plots of virial equation of $\mathrm{C}_{2} \mathrm{H}_{2}$ and $\mathrm{CO}_{2}$ in FJU-6-TATB. 


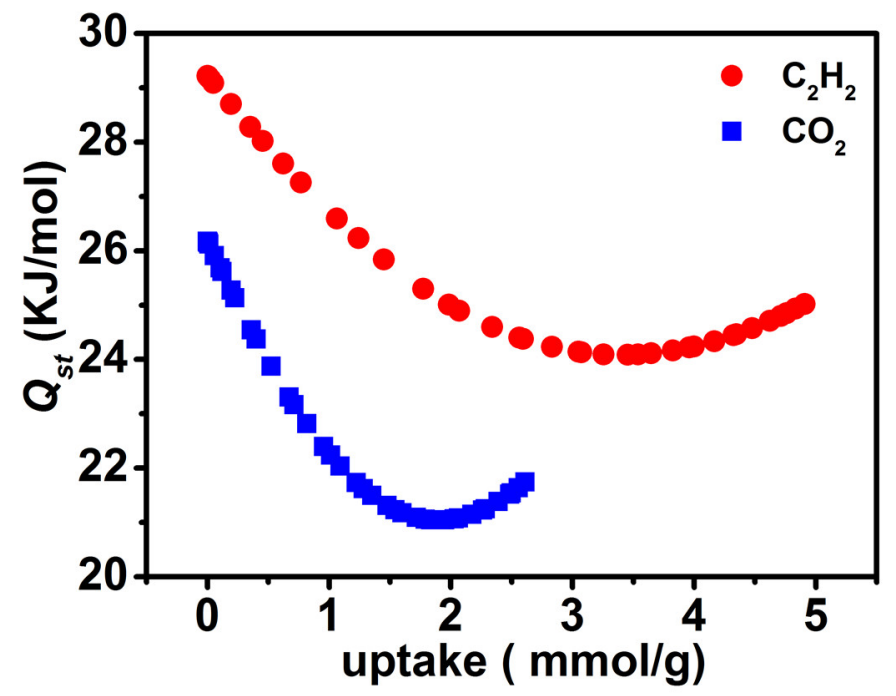

Figure S16. The isosteric heat of $\mathrm{C}_{2} \mathrm{H}_{2}$ and $\mathrm{CO}_{2}$ in FJU-6-TATB.
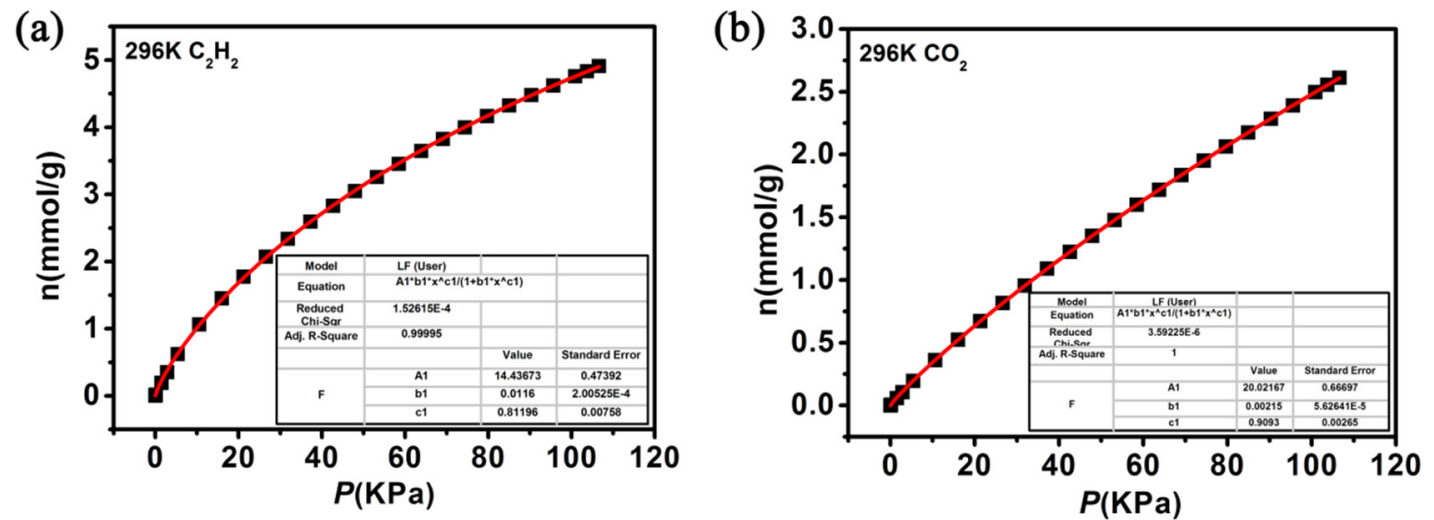

Figure S17. The graphs of the Single-site Langmuir-Freundlich equations fit for adsorption of $\mathrm{C}_{2} \mathrm{H}_{2}(\mathrm{a})$ and $\mathrm{CO}_{2}$ (b) on FJU-6-TATB at $296 \mathrm{~K}$. 
Table S3. Summary of MOFs with $\mathrm{C}_{2} \mathrm{H}_{2} / \mathrm{CO}_{2}$ (50/50) breakthrough performance and related gas uptake and separation values under ambient conditions.

\begin{tabular}{|c|c|c|c|c|c|c|}
\hline Material name & BET & $\begin{array}{c}\mathrm{C}_{2} \mathrm{H}_{2} \text { uptake } \\
\left(\mathrm{cm}^{3} / \mathrm{g}\right)\end{array}$ & $\begin{array}{c}\mathrm{CO}_{2} \text { uptake } \\
\left(\mathrm{cm}^{3} / \mathrm{g}\right)\end{array}$ & $\begin{array}{c}\text { IAST } \\
\text { Selectivity }\end{array}$ & $\begin{array}{l}\text { Separation } \\
\text { factor }^{\mathrm{a}}\end{array}$ & ref \\
\hline HKUST-1 & 1401 & 201 & 113 & 11.0 & - & $\mathrm{S} 12$ \\
\hline JNU-1 & 818 & 27.4 & 4.1 & $285.6-6.6$ & - & S13 \\
\hline SNNU-45 & 1006 & 134.0 & 97.0 & $8.5-4.5$ & $2.9^{\mathrm{b}}$ & S14 \\
\hline JXNU-5 & 406 & 55.9 & 34.8 & 6.0 & $9.9^{\mathrm{b}}$ & $\mathrm{S} 15$ \\
\hline JCM-1 & 550 & 75.0 & 38.0 & 13.7 & $4.4^{\mathrm{c}}$ & S16 \\
\hline NKMOF-1-Ni & 382 & 61.0 & 51.1 & $249.3-30$ & $2.6^{\mathrm{d}}$ & S17 \\
\hline FJU-36a & 409 & 52.2 & 35.5 & 2.8 & $2.1^{\mathrm{e}}$ & S7 \\
\hline FJU-89a & 774 & 101.4 & 61.1 & $4.3-6.6$ & $3.0^{\mathrm{f}}$ & $\mathrm{S} 18$ \\
\hline FJU-90a & 1572 & 180.0 & 103.0 & $6.4-4.3$ & - & S19 \\
\hline UTSA-300a & 311 & 68.9 & 3.3 & $0.02-743$ & - & $\mathrm{S} 20$ \\
\hline ZJU-196 & - & 83.5 & 8.5 & - & - & $\mathrm{S} 21$ \\
\hline UCP-110 & 1384 & 73.4 & 24.3 & $3.7-5.1$ & - & $\mathrm{S} 22$ \\
\hline HOF-3a & 165 & 47.0 & 21.0 & 14.0 & $2.0^{\mathrm{g}}$ & $\mathrm{S} 23$ \\
\hline DICRO-4-Ni-i & 398 & 43 & 23 & 13.9 & $13^{\mathrm{h}}$ & $\mathrm{S} 24$ \\
\hline TIFSIX-2-Cu-i & 685 & 91.8 & 96.3 & 6.5 & $50^{\mathrm{h}}$ & $\mathrm{S} 25$ \\
\hline SIFSIX-3-Ni & 223 & 73.9 & 60.5 & 7.7 & $90^{\mathrm{h}}$ & S25 \\
\hline ZJU-60a & 1627 & 178.7 & 97.7 & - & - & S26 \\
\hline UTSA-74a & 830 & 108.2 & 70.9 & $20-9$ & $20.1^{\mathrm{b}}$ & S27 \\
\hline PCP-33 & 1248 & 121.8 & 58.6 & $6-10$ & - & S28 \\
\hline FeNi-M'MOF & 383 & 96.1 & 60.9 & 24 & $1.7^{\mathrm{b}}$ & S29 \\
\hline UPC-200(Al)-F-BIM & - & 144.5 & 55.5 & 3.1 & - & $\mathrm{S} 30$ \\
\hline MUF-17 & 247 & 67.4 & 56.2 & 6.01 & - & S31 \\
\hline CPM-107op & 319 & 97.5 & 35 & 5.7 & - & S32 \\
\hline \multirow[t]{2}{*}{ FJU-22a } & 828 & 114.8 & 111.3 & - & $1.9^{\mathrm{i}}$ & S33 \\
\hline & & & S22 & & & \\
\hline
\end{tabular}


ZJUT-2a

FJU-6-TATB

a: Separation factor from breakthrough experiments; b: $2 \mathrm{~mL} \mathrm{~min}^{-1}$; c: $7 \mathrm{~mL} \mathrm{~min}^{-1}$; d: $3.2 \mathrm{~mL} \mathrm{~min}^{-1}$; e: 3.6 $\mathrm{mL} \min ^{-1}\left(\mathrm{C}_{2} \mathrm{H}_{2} / \mathrm{CO}_{2} / \mathrm{He}\right.$ 5:5:90); f: $7 \mathrm{~mL} \min ^{-1}\left(\mathrm{C}_{2} \mathrm{H}_{2} / \mathrm{CO}_{2} / \mathrm{He}\right.$ 5:5:90); g: $5 \mathrm{~mL} \min ^{-1} ; \mathrm{h}: 10$

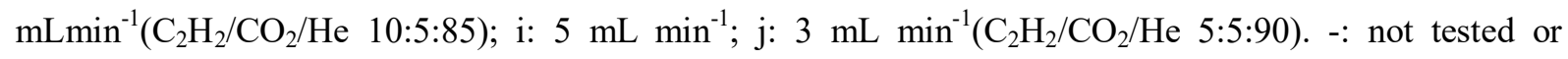
provided.

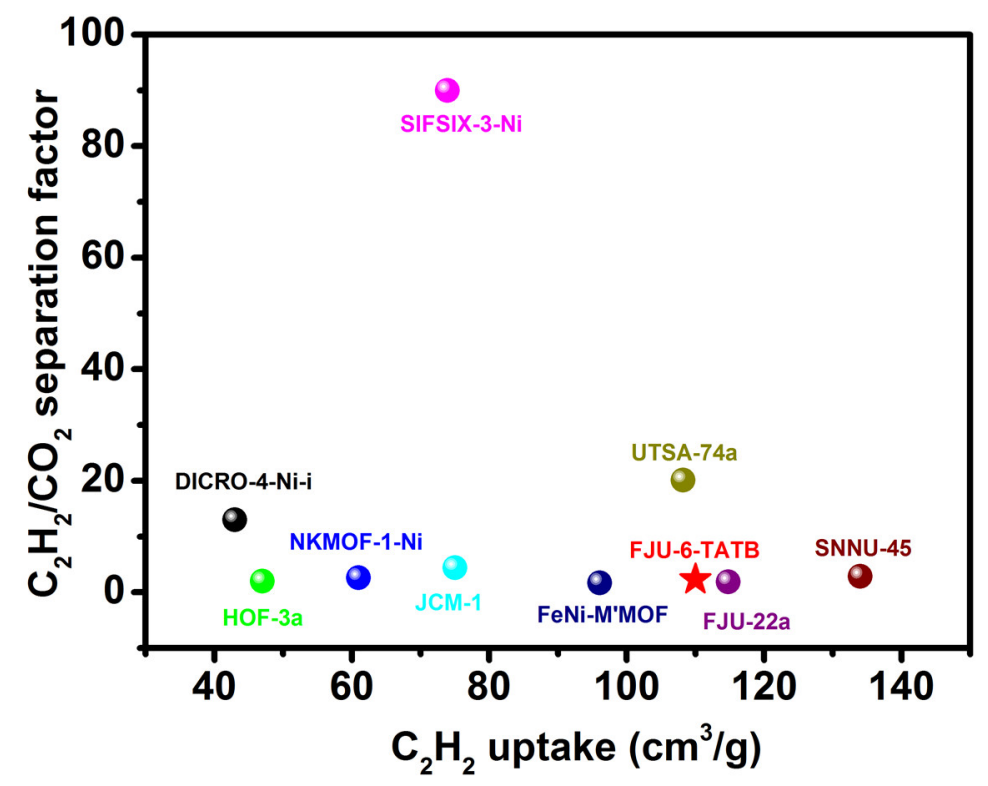

Figure S18. Comparison of $\mathrm{C}_{2} \mathrm{H}_{2} / \mathrm{CO}_{2}$ separation factor and $\mathrm{C}_{2} \mathrm{H}_{2}$ uptake at 1 bar in FJU-6-TATB,

FJU-6-TATB and other porous materials under ambient conditions. (Experimental conditions are shown in Table S3.) 


\section{References}

(1) Dolomanov, A. V.; Bourhis, L. J.; Gildea, R. J.; Howard, J. A. K. and Puschmann, H. OLEX2: a complete structure solution, refinement and analysis program. J. Appl. Crystallogr., 2009, 42, 339-341.

(2) Palatinus, L. and Chapuis, G. SUPERFLIP-a computer program for the solution of crystal structures by charge flipping in arbitrary dimensions. J. Appl. Crystallogr. 2007, 40,786-790.

(3) Sheldrick, G. M. A short history of SHELX. Acta Crystallogr., Sect. A: Found. Crystallogr.2008, 64, $112-122$

(4) Sarkisov, L. and Harrison, A. Computational structure characterisation tools in application to ordered and disordered porous materials. Mol. Simul. 2011, 37, 1248-1257.

(5) Spek, A. L. Single-crystal structure validation with the program PLATON. J. Appl. Crystallogr. 2003, 36, 7-13.

(6) Myers, A. L.; Prausnitz, J. M. Thermodynamics of mixed-gas adsorption. AIChE J. 1965, 11, 121-127.

(7) Liu, L. Z.; Yao, Z. Z.; Ye, Y. X.; Chen, L. J.; Lin, Q. J.; Yang, Y. S.; Zhang, Z. J. and Xiang, S. C. Robustness, Selective Gas Separation, and Nitrobenzene Sensing on Two Isomers of Cadmium Metal-Organic Frameworks Containing Various Metal-O-Metal Chains, Inorg. Chem. 2018, 57, 12961-12968.

(8) Chang, H.; Wu, Z. X. Experimental study on adsorption of carbon dioxide by 5A molecular sieve for helium purification of high-temperature gas-cooled reactor. Ind. Eng. Chem. Res. 2009, 48, 4466-4473.

(9) Liu, J.; Tian, J.; Thallapally, P. K.; McGrail, B. P. Selective $\mathrm{CO}_{2}$ capture from flue gas using metal-organic frameworks-a fixed bed study. J. Phys.Chem. C. 2012, 116, 9575-9581.

(10) Yang, J. X.; Tao, X. T.; Yuan, C. X.; Yan, Y. X.; Wang, L.; Liu, Z.; Ren, Y.; Jiang, M. H. A facile synthesis and properties of multicarbazole molecules containing multiple vinylene bridges. J. Am. Chem. Soc. 2005, 127, 3278-3279.

(11) Yao, Q.; Gómez, A. B.; Su, J.; Pascanu, V.; Yun, Y.; Zheng, H.; Chen, H.; Liu, L.; Abdelhamid, H. N.; Martín-Matute, B.; Zou, X.; Series of highly stable isoreticular lanthanide metal-organic frameworks with expanding pore size and tunable luminescent properties. Chem. Mater. 2015, 27, 5332-5339.

(12) Xiang, S.; Zhou, W.; Gallegos, J. M.; Liu, Y. and Chen, B. Exceptionally High Acetylene Uptake in a Microporous Metal-Organic Framework with Open Metal Sites. J. Am. Chem. Soc., 2009, 131, 12415-12419.

(13) Zeng, H.; Xie, M.; Huang, Y. L.; Zhao, Y.; Xie, X. J.; Bai, J. P.; Wan, M. Y.; Krishna, R.; Lu, W.; Li, D. Induced Fit of $\mathrm{C}_{2} \mathrm{H}_{2}$ in a Flexible MOF through Cooperative Action of Open Metal Sites. Angew. Chem., Int. Ed. 2019, 58, 8515-8519.

(14) Li, Y. P.; Wang, Y.; Xue, Y. Y.; Li, H. P.; Zhai, Q. G.; Li, S. N.; Jiang, Y. C.; Hu, M. C. and Bu, X. H. Ultramicroporous Building Units as a Path to Bi-microporous Metal-Organic Frameworks with High Acetylene Storage and Separation Performance, Angew. Chem. Int. Ed. 2019, 131, 13724-13729.

(15) Liu, R.; Liu, Q. Y.; Krishna, R.; Wang, W.; He, C. T.; Wang, Y. L. Water-Stable Europium 1,3,6,8-Tetrakis(4-carboxylphenyl)pyrene Framework for Efficient $\mathrm{C}_{2} \mathrm{H}_{2} / \mathrm{CO}_{2}$ Separation. Inorg. Chem. 2019, 58, 5089-5095.

(16) Lee, J.; Chuah, C.Y.; Kim, J.; Kim, Y.; Ko, N.; Seo, Y.; Kim, K.; Bae, T. H.; Lee, E.; Separation of Acetylene from Carbon Dioxide and Ethylene by a Water-Stable Microporous Metal-Organic Framework with Aligned Imidazolium Groups inside the Channels. Angew. Chem., Int. Ed. 2018, 57, 7869-7873.

(17) Peng, Y. L.; Pham, T.; Li, P.; Wang, T.; Chen, Y.; Chen, K. J.; Forrest, K. A.; Space, B.; Cheng, P.; Zaworotko, M. J.; Zhang, Z. Robust Ultramicroporous Metal-Organic Frameworks with Benchmark Affinity for Acetylene. Angew. Chem., Int. Ed. 2018, 57, 10971-10975.

(18) Ye, Y.; Chen, S.; Chen, L.; Huang, J.; Ma, Z.; Li, Z.; Yao, Z. ; Zhang, J.; Zhang, Z.; Xiang, S.; Additive-Induced Supramolecular Isomerism and Enhancement of Robustness in Co(II)-Based MOFs for Efficiently Trapping Acetylene from Acetylene-Containing Mixtures. ACS Appl. Mater. Interfaces 2018, 10, 30912-30918.

(19) Ye, Y. X.; Ma, Z. L.; Lin, R. B.; Krishna, R.; Zhou, W.; Lin, Q. J.; Zhang, Z. J.; Xiang, S. C. and Chen, B. L. Pore Space Partition within a Metal-Organic Framework for Highly Efficient $\mathrm{C}_{2} \mathrm{H}_{2} / \mathrm{CO}_{2}$ Separation. $J$. Am. Chem. Soc. 2019, 141, 4130-4136.

(20) Lin, R. B.; Li, L.; Wu, H.; Arman, H.; Li, B.; Lin, R.-G.; Zhou, W.; Chen, B. Optimized Separation of Acetylene from Carbon Dioxide and Ethylene in a Microporous Material. J. Am. Chem. Soc. 2017, 139, 8022-8028. 
(21) Zhang, L.; Jiang, K.; Li, L.; Xia, Y. P.; Hu, T. L.; Yang, Y.; Cui, Y. ; Li, B.; Chen, B.; Qian, G.; Efficient separation of $\mathrm{C}_{2} \mathrm{H}_{2}$ from $\mathrm{C}_{2} \mathrm{H}_{2} / \mathrm{CO}_{2}$ mixtures in an acid-base resistant metal-organic framework. Chem. Commun. 2018, 54, 4846-4849.

(22) Fan, W.; Wang, X.; Liu, X.; Xu, B.; Zhang, X.; Wang, W.; Wang, X.; Wang, Y.; Dai, F.; Yuan, D.; Sun, D.; Regulating $\mathrm{C}_{2} \mathrm{H}_{2}$ and $\mathrm{CO}_{2}$ Storage and Separation through Pore Environment Modification in a Microporous Ni-MOF. Acs Sustain Chem. Eng. 2019, 7, 2134-2140.

(23) Li, P.; He, Y.; Zhao, Y.; Weng, L.; Wang, H.; Krishna, R.; Wu, H.; Zhou, W.; OKeeffe, M.; Han, Y.; and Chen, B. A Rod-Packing Microporous Hydrogen-Bonded Organic Framework for Highly Selective Separation of $\mathrm{C}_{2} \mathrm{H}_{2} / \mathrm{CO}_{2}$ at Room Temperature, Angew. Chem. 2015, 127, $584-587$.

(24) Scott, H. S.; Shivanna, M.; Bajpai, A.; Madden, D. G.; Chen, K.; Pham, T.; Forrest, K. A.; Hogan, A.; Space, B.; Perry IV, J. J. and Zaworotko, M. J. Benchmark $\mathrm{C}_{2} \mathrm{H}_{2} / \mathrm{CO}_{2}$ and $\mathrm{CO}_{2} / \mathrm{C}_{2} \mathrm{H}_{2}$ Separation by Two Closely Related Hybrid Ultramicroporous Materials. ACS Appl. Mater. Interfaces 2017, 9, 33395-33400.

(25) Chen, K. J.; Scott, H. S.; Madden, D. G.; Pham, T.; Kumar, A.; Bajpai, A.; Lusi, M.; Forrest, K. A.; Space, B.; Perry IV, J. J.; Zaworotko, M. J. Benchmark $\mathrm{C}_{2} \mathrm{H}_{2} / \mathrm{CO}_{2}$ and $\mathrm{CO}_{2} / \mathrm{C}_{2} \mathrm{H}_{2}$ Separation by Two Closely Related Hybrid Ultramicroporous Materials. Chem. 2016, 1, 753- 765.

(26) Duan, X.; Zhang, Q.; Cai, J.; Yang, Y.; Cui, Y.; He, Y.; Wu, C.; Krishna, R.; Chen, B.; Qian, G.; A new metal-organic framework with potential for adsorptive separation of methane from carbon dioxide, acetylene, ethylene, and ethane established by simulated breakthrough experiments. J. Mater. Chem. A 2014, 2, 2628-2633.

(27) Luo, F.; Yan, C.; Dang, L.; Krishna, R.; Zhou, W.; Wu, H.; Dong, X.; Han, Y.; Hu, T. L.; O’Keeffe, M.; Wang, L.; Luo, M.; Lin, R. B.; and Chen, B. UTSA-74: A MOF-74 Isomer with Two Accessible Binding Sites per Metal Center for Highly Selective Gas Separation, J. Am. Chem. Soc. 2016, 138, 5678-5684.

(28) Duan, J.; Jin, W. and Krishna, R. Natural Gas Purification Using a Porous Coordination Polymer with Water and Chemical Stability, Inorg. Chem. 2015, 54, 4279-4284

(29) Gao, J.; Qian, X.; Lin, R. B.; Krishna, R; Wu, H; Zhou, W. and Chen, B.; Mixed Metal-Organic Framework with Multiple Binding Sites for Efficient $\mathrm{C}_{2} \mathrm{H}_{2} / \mathrm{CO}_{2}$ Separation. Angew. Chem. Int. Ed. 2020, 59, $4396-4400$.

(30) Fan, W., Yuan, S., Wang, W., Feng, L., Liu, X., Zhang, X., Wang X., Kang, Z., Dai, F., Yuan, D., Sun, D. and Zhou, H. C. Optimizing Multivariate Metal-Organic Frameworks for Efficient $\mathrm{C}_{2} \mathrm{H}_{2} / \mathrm{CO}_{2}$ Separation J. Am. Chem. Soc. 2020, DOI: 10.1021/jacs.0c00805.

(31) Qazvini, O. T.; Babarao, R. and Telfer, S. G. Multipurpose Metal-Organic Framework for the Adsorption of Acetylene: Ethylene Purification and Carbon Dioxide Removal. Chem. Mater, 2019, 31, 4919-4926.

(32) Yang, H.; Trieu, T. X.; Zhao, X.; Wang, Y.; Wang, Y.; Feng, P. and Bu, X.; Lock-and-Key and Shape-Memory Effects in an Unconventional Synthetic Path to Magnesium Metal-Organic Frameworks, Angew. Chem. Int. Ed. 2019, 58, 11757-11762.

(33) Yao, Z.; Zhang, Z.; Liu, L.; Li, Z.; Zhou, W.; Zhao, Y.; Han, Y.; Chen, B.; Krishna, R. and Xiang, S. Extraordinary Separation of Acetylene-Containing Mixtures with Microporous Metal-Organic Frameworks with Open O Donor Sites and Tunable Robustness through Control of the Helical Chain Secondary Building Units, Chem. Eur. J. 2016, 22, 5676 - 5683.

(34) Wen, H.; Liao, C.; Li, L.; Yang, L.; Wang, J.; Huang, L.; Li, B.; Chen, B. and Hu, J. Reversing $\mathrm{C}_{2} \mathrm{H}_{2}-$ $\mathrm{CO}_{2}$ adsorption selectivity in an ultramicroporous metal-organic framework platform, Chem. Commun, 2019, 55, 11354-11357. 